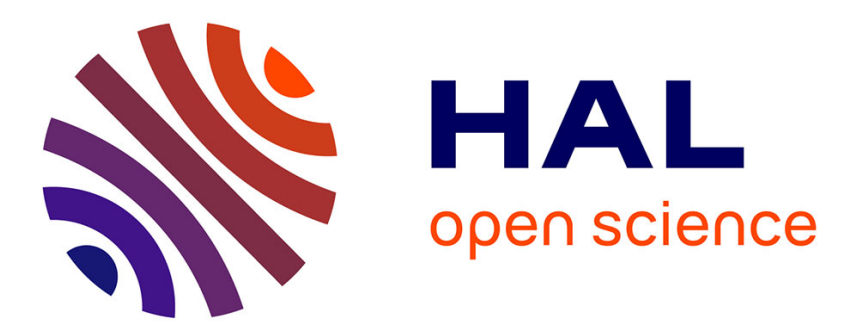

\title{
Convergence analysis of a penalization method for the three-dimensional motion of a rigid body in an incompressible viscous fluid
}

\author{
Claire Bost, Georges-Henri Cottet, Emmanuel Maitre
}

\section{To cite this version:}

Claire Bost, Georges-Henri Cottet, Emmanuel Maitre. Convergence analysis of a penalization method for the three-dimensional motion of a rigid body in an incompressible viscous fluid. SIAM Journal on Numerical Analysis, 2010, 48 (4), pp.1313-1337. 10.1137/090767856 . hal-00352808v2

\section{HAL Id: hal-00352808 \\ https://hal.science/hal-00352808v2}

Submitted on 12 Jul 2010

HAL is a multi-disciplinary open access archive for the deposit and dissemination of scientific research documents, whether they are published or not. The documents may come from teaching and research institutions in France or abroad, or from public or private research centers.
L'archive ouverte pluridisciplinaire HAL, est destinée au dépôt et à la diffusion de documents scientifiques de niveau recherche, publiés ou non, émanant des établissements d'enseignement et de recherche français ou étrangers, des laboratoires publics ou privés. 


\title{
Convergence analysis of a penalization method for the three-dimensional motion of a rigid body in an incompressible viscous fluid
}

\author{
C. Bost, G.-H. Cottet and E. Maitre \\ Université de Grenoble and CNRS, Laboratoire Jean Kuntzmann \\ BP 53, 38041 Grenoble Cedex 9, France
}

May 19, 2010

\begin{abstract}
We present and analyze a penalization method which extends the the method of [2] to the case of a rigid body moving freely in an incompressible fluid. The fluid-solid system is viewed as a single variable density flow. The interface is captured by a color function satisfying a transport equation. The solid velocity is computed by averaging at every time the flow velocity in the solid phase. This velocity is used to penalize the flow velocity at the fluid-solid interface and to move the interface. Numerical illustrations are provided to illustrate our convergence result. A discussion of our result in the light of existing existence results is also given.
\end{abstract}

\section{Introduction}

In this paper we are concerned with the numerical analysis of a penalization method for the twoway interaction of a rigid body with an incompressible fluid in three dimensions. The traditional numerical approach to deal with fluid-structure problems is the so-called ALE (for Arbitrary Lagrangian Eulerian) method where fluids (resp solids) are described in an Eulerian (resp Lagrangian) framework. Fluids are computed on a moving mesh fitting the solids and stress and velocity continuity are used to derive the appropriate boundary conditions on the fluid/solid interface [17].

Another way is to use a fixed mesh and rely on a Lagrange-Galerkin approach where the fluid is solved using a characteristics-finite-element method in a space where the velocity field is rigid in the solid, and the solid itself moved by a Lagrangian equation. In [19] the convergence of a scheme following this approach is proved in the case where the densities of fluid and structure are equal.

Alternate methods can be devised where the whole fluid-solid system is seen as a multiphase flow and the fluid/solid interface is captured implicitly rather than explicitly. Likewise, the interface continuity conditions are recovered in an implicit fashion. The rigid motion inside the solid 
phase can be enforced through a Lagrange multiplier [11]. The method we consider here is of this type but the rigid motion is approximately satisfied in the solid through penalization.

Penalization methods have already been considered in the past for this problem. In [6] the authors considered a single solid ball and worked inside the frame moving with its center. Then they penalized the mean velocity of the (virtual) fluid inside this ball. Their method is restricted to one ball. $\operatorname{In}[20,15]$ the penalization is applied to the deformation tensor inside the body. In [20] this method is used to prove the existence of solutions for the fluid-solid interaction variational problem in two dimensions. In [15] it is used together with a two-dimensional finite element method in a variational framework. Here the penalization is applied to the flow velocity itself. The method thus extends the one devised and analyzed in [2] in the case of a rigid solid with prescribed motion.

In our method the determination of the body velocity is part of the problem. This velocity, instead of the flow velocity, is used to move the solid phase. This has a crucial practical importance, in particular for problems with large displacements and strong shear, since it ensures that the solid remains rigid at the discrete level, although the rigidity constraint in the flow field is only approximately satisfied. A vorticity formulation of the method and its validation on a number of $2 \mathrm{D}$ and $3 \mathrm{D}$ reference cases are given in [7]. An outline of the paper is as follows. In section 2 we recall the weak formulation of the problem and we describe the penalization method. Section 3 is devoted to the convergence proof. In section 4 we provide some numerical illustrations. Section 5 is devoted to some concluding remarks. The proofs of some technical results used in section 3 are given in the appendix.

\section{Weak formulation and penalized problem}

Let $\Omega$ be an open bounded domain of $\mathbb{R}^{3}$, filled with a viscous incompressible and homogeneous fluid of density $\rho_{f}>0$ and viscosity $\mu>0$. Inside this domain, we consider the motion of an immersed homogeneous rigid solid of density $\rho_{s}>0$ during a time interval $[0, T], T>0$, chosen so that the solid never comes in contact with $\partial \Omega$. For $t \in[0, T]$, we denote by $\Omega_{f}(t)$ and $\Omega_{s}(t)$ the non-empty fluid and solid open connected domains, with $\overline{\Omega_{s}(t)} \cup \overline{\Omega_{f}(t)}=\bar{\Omega}$ and $\Omega_{f}(t) \cap \Omega_{s}(t)=\emptyset$. The center of mass of the solid is denoted by $x_{G}(t)$, its mass and inertia tensor by $M$ and $J(t)$. 
Without loss of generality we assume that $M=1$. Then

$$
x_{G}(t)=\int_{\Omega_{s}(t)} \rho_{s} x d x, \quad J(t)=\int_{\Omega_{s}(t)} \rho_{s}\left(r^{2} \mathbb{I}-r \otimes r\right) d x
$$

where $r(x, t)=x-x_{G}(t)$. The system is subject to a body density force $g$ (usually gravity).

\subsection{Weak formulation}

The basic formulation of this fluid-solid coupling is the following : given initial conditions,

$$
x_{G}^{\prime}(0)=v_{g}^{0}, \quad \omega_{u}(0)=\omega_{u}^{0}, \quad u=u^{0}, \quad \Omega_{s}(0)=\Omega_{s}^{0}
$$

supplemented with

$$
x_{G}(0)=\int_{\Omega_{s}^{0}} x d x, \quad X_{s}(x, 0)=x,
$$

find $t \rightarrow \Omega_{s}(t)$ and $(x, t) \rightarrow(u(x, t), p(x, t))$ solution for $t>0$ of

$$
\begin{array}{rr}
\rho_{f}\left(u_{t}+(u \cdot \nabla) u\right)-2 \mu \operatorname{div}(D(u))+\nabla p=\rho_{f} g & \text { on } \Omega_{f}(t), \quad(3) \\
\operatorname{div} u=0 & \text { on } \Omega_{f}(t), \quad(4) \\
u=0 & \text { on } \partial \Omega, \quad(5) \\
u=x_{G}^{\prime}+\omega_{u} \times r & \text { on } \partial \Omega_{s}(t),(6) \\
x_{G}^{\prime \prime}(t)=g+\int_{\partial \Omega_{s}(t)}(\Sigma n) d s, & \\
J(t) \omega_{u}^{\prime}(t)=-\omega_{u}(t) \times\left(J(t) \omega_{u}(t)\right)+\int_{\Omega_{s}(t)} \rho_{s}(r \times g) d x+\int_{\partial \Omega_{s}(t)} r \times(\Sigma n) d s, \\
\Omega_{s}(t)=X_{s}\left(t, \Omega_{s}^{0}\right),
\end{array}
$$

where $n$ denotes the unit outward normal on $\partial \Omega_{s}(t)$, and $\Sigma$ is the fluid stress tensor.

In this formulation the last two equations describe the rigid motion of $\Omega_{s}(t)$. In order to give a weak formulation of this problem, let us introduce some function spaces. From now on, $u$ will denote the velocity field on the whole computational domain $\Omega$. We define

$$
\mathcal{V}=\left\{u \in H_{0}^{1}(\Omega), \operatorname{div} u=0\right\}, \quad \mathcal{H}=\left\{u \in L^{2}(\Omega), \operatorname{div} u=0, u \cdot n=0 \text { on } \partial \Omega\right\}
$$

and, with the notations of [20] extended to the three dimensional case,

$$
\mathcal{K}(t)=\left\{u \in \mathcal{V}, D(u)=0 \text { in } \Omega_{s}(t)\right\}
$$

$$
=\left\{u \in \mathcal{V}, \exists\left(V_{u}, \omega_{u}\right) \in \mathbb{R}^{3} \times \mathbb{R}, u=V_{u}+\omega_{u} \times r \text { in } \Omega_{s}(t)\right\} .
$$


Next we define a density on the whole domain by setting $\rho=\rho_{s} \chi_{\Omega_{s}(t)}+\rho_{f} \chi_{\Omega_{f}(t)}$, where $\chi_{A}$ denotes the characteristic function of set $A$, which takes value 1 inside $A$ and 0 outside. Let us note $Q=\Omega \times] 0, T[$. Then the weak formulation is the following [14]: given initial conditions $H^{0}=\chi_{\Omega_{s}^{0}}, \rho^{0}=\rho_{s} H^{0}+\rho_{f}\left(1-H^{0}\right)$ and $u=u^{0} \in \mathcal{K}(0)$, find $(x, t) \rightarrow(\rho(x, t), u(x, t), H(x, t))$ such that

$$
\left\{\begin{array}{l}
u \in L^{\infty}(0, T, \mathcal{H}) \cap L^{2}(0, T, \mathcal{V}), \quad H, \rho \in \mathcal{C}\left(0, T ; L^{q}(\Omega)\right) \forall q \geq 1, \\
u(t) \in \mathcal{K}(t) \text { for a.e. } t \in] 0, T\left[, \text { with } \Omega_{s}(t)=\{x \in \Omega, H(x, t)=1\},\right. \\
\forall \xi \in H^{1}(Q) \cap L^{2}(0, T ; \mathcal{K}(t)), \\
\quad \int_{\Omega}\left[\rho u \cdot \partial_{t} \xi+(\rho(u \cdot \nabla) u-2 \mu D(u)): D(\xi)+\rho g \cdot \xi\right] d x=\frac{d}{d t} \int_{\Omega} \rho u \cdot \xi d x, \\
\forall \psi \in \mathcal{C}^{1}(Q), \psi(T)=0, \\
\quad \int_{0}^{T} \int_{\Omega}^{T} H \frac{\partial \psi}{\partial t}+H u \cdot \nabla \psi d x d t+\int_{\Omega} H^{0} \psi(0) d x=0 \\
\quad \int_{0}^{T} \int_{\Omega}^{\rho} \rho \frac{\partial \psi}{\partial t}+\rho u \cdot \nabla \psi d x d t+\int_{\Omega} \rho^{0} \psi(0) d x=0 .
\end{array}\right.
$$

Note that we could equivalently have defined $\rho=\rho_{s} H+\rho_{f}(1-H)$, as $\rho^{0}$ is piecewise constant, and transported by the same velocity field than $H$.

\subsection{Penalized problem}

For $\eta>0$, we consider the following penalized problem:

given $\left(\rho_{\eta}(0)=\rho^{0}, u_{\eta}(0)=u_{\eta}^{0}, H_{\eta}(0)=\chi_{\Omega_{s}^{0}}\right)$, to find $\left(\rho_{\eta}, u_{\eta}, p_{\eta}, H_{\eta}\right)$, with

$\rho_{\eta}, H_{\eta} \in L^{\infty}(] 0, T[\times \Omega), \quad u_{\eta} \in L^{\infty}(0, T ; \mathcal{H}) \cap L^{2}(0, T ; \mathcal{V}), \quad p_{\eta} \in L^{2}(Q), \quad \frac{\partial}{\partial t}\left(\rho_{\eta} u_{\eta}\right) \in L^{2}\left(0, T ; \mathcal{V}^{\prime}\right)$

solution in the sense of distributions in $Q$ of

$$
\begin{array}{r}
\frac{\partial\left(\rho_{\eta} u_{\eta}\right)}{\partial t}+\operatorname{div}\left(\rho_{\eta} u_{\eta} \otimes u_{\eta}\right)-2 \mu \operatorname{div}\left(D\left(u_{\eta}\right)\right)+\nabla p_{\eta}+\frac{1}{\eta} \rho_{\eta} H_{\eta}\left(u_{\eta}-u_{\eta, s}\right)=\rho_{\eta} g \\
\operatorname{div} u_{\eta}=0 \\
u_{\eta, s}=\frac{1}{M_{\eta}} \int_{\Omega} \rho_{\eta} u_{\eta} H_{\eta} d x+\left(J_{\eta}^{-1} \int_{\Omega} \rho_{\eta}\left(r_{\eta} \times u_{\eta}\right) H_{\eta} d x\right) \times r_{\eta} \\
\rho_{\eta_{t}}+u_{\eta} . \nabla \rho_{\eta}=0 \\
H_{\eta_{t}}+u_{\eta, s} . \nabla H_{\eta}=0
\end{array}
$$

We set $\Omega_{s}^{\eta}(t)=\left\{x \in \Omega, H_{\eta}(x, t)=1\right\}$. In equation (14) we divided the first term by $M_{\eta}=$ $\int_{\Omega} \rho_{\eta} H_{\eta} d x$, which is not constant in time in general. On contrary we have $\left|\Omega_{s}^{\eta}(t)\right|=\int_{\Omega} H_{\eta} d x=$ $\left|\Omega_{s}^{0}\right|$ since $u_{\eta, s}$ is divergence free and $H_{\eta}$ vanishes on $\partial \Omega$ (we assumed no contact of the solid with 
$\partial \Omega)$. The inertia tensor is defined as

$$
J_{\eta}=\int_{\Omega} \rho_{\eta} H_{\eta}\left(r_{\eta}^{2} \mathbb{I}-r_{\eta} \otimes r_{\eta}\right) d x=\int_{\Omega_{s}^{\eta}(t)} \rho_{\eta}\left(r_{\eta}^{2} \mathbb{I}-r_{\eta} \otimes r_{\eta}\right) d x
$$

with $r_{\eta}=x-x_{G \eta}=x-\int_{\Omega} \rho_{\eta} H_{\eta} x d x$.

For $a \in \mathbb{R}^{3} \backslash\{0\}, a^{T} J_{\eta} a=\int_{\Omega_{s}^{\eta}(t)} \rho_{\eta}\left|r_{\eta} \times a\right|^{2} d x \geq \min \left(\rho_{s}, \rho_{f}\right) \int_{\Omega_{s}^{\eta}(t)}\left|r_{\eta} \times a\right|^{2} d x$ (see estimate (18)). This last quantity being strictly positive for an open nonempty integration set, $J_{\eta}$ is nonsingular (we recall that $\left|\Omega_{s}^{\eta}(t)\right|=\left|\Omega_{s}^{0}\right|>0$ ).

Before stating our convergence result, a few remarks are in order.

First one may wonder about the well-posedness of the above problem. However it will directly result from the a priori estimates and convergence arguments given in the following that this problem does have at least a weak solution. Indeed, these arguments could easily be used to show the convergence of the solutions to a linearized version - or finite-dimension approximation - of (12)-(16). Next we can observe that in this model we penalize the difference between $u_{\eta}$ and the projection of $u_{\eta}$ onto velocity fields rigid in the solid domain, namely $u_{\eta, s}$ (see lemma 3.1 below). The density is transported with the original velocity field so that estimates on the Navier-Stokes equations are easier to obtain. The characteristic function is transported by the rigid velocity so that the shape of $\Omega_{s}^{\eta}(t)$ remains undeformed (this is exactly the Eulerian counterpart of (910)). As observed in [7] this has a practical importance (in particular it means that the rigid solid can be recovered exactly through simple algebra from its initial shape). As far as numerical analysis is concerned, it also provides "for free" regularity properties on the computed rigid body, as soon as the initial body is smooth. The price to pay is that the level sets of $\rho_{\eta}$ and $H_{\eta}$ do not coincide, i.e. in general we do not have $\rho_{\eta} H_{\eta}=\rho_{s}$ as in the non penalized formulation. Note also that in principle we should prescribe a boundary value for $H_{\eta}$ on $\partial \Omega$ when $u_{\eta, s}$ is inward. Since our analysis is restricted to times when the solid body does not approach the boundary of the computational box, we can take this boundary value to be zero, which amounts to solve (16) on $\mathbb{R}^{n}$ and take its restriction to $\Omega$.

Let us now comment on the differences between the present method and the methods in $[20,18,22]$. The method used in [20] to prove the existence of the fluid-body problem uses a penalization acting on the deformation. It does not require to compute the rigid body velocity $u_{\eta, s}$. However it does not guarantees a strictly rigid transport of the body, which is clearly a 
drawback form a practical point of view. The projection method in $[18,22]$ can be seen as a particular discretization of the present penalization method, with an explicit time-discretization of the penalization term. In practice this result in a limitation of the penalization coefficient which has some We will come back to this point in our numerical illustrations in section 4 . Numerical comparisons for a benchmark challenging $3 \mathrm{D}$ case are also given in [7].

In the following sections we will prove the convergence of at least a subsequence of $\left(\rho_{\eta}, u_{\eta}, p_{\eta}, H_{\eta}\right)$ to the weak solution defined above. Next section starts with some a priori estimates which will provide weak convergence of subsequences. In section 3.3 we will have to use more sophisticated tools adapted from [20] to get some strong convergence in $u_{\eta}$ which will allow us to pass to the limit in nonlinear terms of $\left(P_{\eta}\right)$. More precisely we prove the following result.

Theorem 2.1. Under the regularity assumptions of section 2, let $\left(\rho_{\eta}, u_{\eta}, p_{\eta}, H_{\eta}\right)$ a solution of $\left(P_{\eta}\right)$. Then there exists a subsequence of $\left(\rho_{\eta}, u_{\eta}, H_{\eta}\right)$ and functions $(\rho, u, H)$ such that

$$
\begin{gathered}
\rho_{\eta} \rightarrow \rho, \quad H_{\eta} \rightarrow H \text { strongly in } C\left(0, T ; L^{q}(\Omega)\right) \text { for all } q \geq 1, \\
u_{\eta} \rightarrow u \text { strongly in } L^{2}(Q) \text { and weakly in } L^{2}\left(0, T ; H_{0}^{1}(\Omega)\right) \cap L^{\infty}\left(0, T ; L^{2}(\Omega)\right)
\end{gathered}
$$

and such that $(\rho, u, H)$, is a solution of (11).

Before proceeding to the proof, let us point out a few remarks. For a sake of simplicity in the notations we have stated our penalization method and theorem for a single rigid body. It will be apparent from the proof below that it readily extends to the case of several bodies. Furthermore, the time to which the convergence result is restricted, is essentially the time for which contact of the rigid body do not touch the boundary of $\Omega$ (in the case of several bodies it would be the time on which we can ensure that contact between bodies do not happen). As a result if we consider periodic boundary conditions and a single body convergence holds for all times.

\section{Proof of theorem 2.1}

The following lemma states that $u_{\eta, s}$, as defined in $\left(P_{\eta}\right)$, is the projection of $u_{\eta}$ onto velocity fields which are rigid on $\Omega_{s}^{\eta}(t)$.

Lemma 3.1. Let $\xi$ be a rigid velocity field, i.e. such that $\xi(x)=V_{\xi}+\omega_{\xi} \times r(x)$ for some constant 
vectors $V_{\xi} \in \mathbb{R}^{3}$ and $\omega_{\xi} \in \mathbb{R}^{3}$. Then if $u_{\eta, s}$ is defined by (14) there holds

$$
\int_{\Omega} \rho_{\eta} H_{\eta}\left(u_{\eta}-u_{\eta, s}\right) \cdot \xi d x=0
$$

Moreover, the result holds if $\xi$ is a time dependent velocity field rigid in $\Omega_{s}^{\eta}(t)$ at time $t$.

Proof. Let the mean translation and angular velocities be defined as

$$
V_{u}=\frac{1}{M_{\eta}} \int_{\Omega} \rho_{\eta} H_{\eta} u_{\eta} d x \quad \omega_{u}=J_{\eta}^{-1} \int_{\Omega} \rho_{\eta} H_{\eta}\left(r_{\eta} \times u_{\eta}\right) d x
$$

then

$$
\begin{aligned}
\int_{\Omega} \rho_{\eta} H_{\eta}\left(u_{\eta}-u_{\eta, s}\right) \cdot \xi d x & =\int_{\Omega} \rho_{\eta} H_{\eta}\left[u_{\eta}-\left(V_{u}+\omega_{u} \times r_{\eta}\right)\right] \cdot\left[V_{\xi}+\omega_{\xi} \times r_{\eta}\right] d x \\
& =V_{\xi} \cdot \int_{\Omega} \rho_{\eta} H_{\eta} u_{\eta} d x+\omega_{\xi} \cdot \int_{\Omega} \rho_{\eta} H_{\eta}\left(r_{\eta} \times u_{\eta}\right) d x-V_{u} \cdot V_{\xi} \int_{\Omega} \rho_{\eta} H_{\eta} d x \\
& -V_{u} \cdot\left(\omega_{\xi} \times \int_{\Omega} \rho_{\eta} H_{\eta} r_{\eta} d x\right)-V_{\xi} \cdot\left(\omega_{u} \times \int_{\Omega} \rho_{\eta} H_{\eta} r_{\eta} d x\right) \\
& -\int_{\Omega} \rho_{\eta} H_{\eta}\left(\omega_{u} \times r_{\eta}\right) \cdot\left(\omega_{\xi} \times r_{\eta}\right) d x \\
& =V_{\xi} \cdot\left(M_{\eta} V_{u}\right)+\omega_{\xi} \cdot\left(J_{\eta} \omega_{u}\right)-V_{u} \cdot\left(M_{\eta} V_{\xi}\right) \\
& -V_{u} \cdot\left(\omega_{\xi} \times \int_{\Omega} \rho_{\eta} H_{\eta} r_{\eta} d x\right)-V_{\xi} \cdot\left(\omega_{u} \times \int_{\Omega} \rho_{\eta} H_{\eta} r_{\eta} d x\right) \\
& -\int_{\Omega} \rho_{\eta} H_{\eta}\left(\omega_{u} \times r_{\eta}\right) \cdot\left(\omega_{\xi} \times r_{\eta}\right) d x .
\end{aligned}
$$

As $\left(\omega_{u} \times r_{\eta}\right) \cdot\left(\omega_{\xi} \times r_{\eta}\right)=\left(\omega_{\xi} \cdot \omega_{u}\right) r_{\eta}^{2}-\left(r_{\eta} \cdot \omega_{\xi}\right)\left(r_{\eta} \cdot \omega_{u}\right)$, we have $\int_{\Omega} \rho_{\eta} H_{\eta}\left(\omega_{u} \times r_{\eta}\right) \cdot\left(\omega_{\xi} \times r_{\eta}\right) d x=$ $\omega_{\xi} \cdot\left(J_{\eta} \omega_{u}\right)$.

Finally, by definition of $r_{\eta}, \int_{\Omega} \rho_{\eta} H_{\eta} r_{\eta} d x=0$, and we get

$$
\int_{\Omega} \rho_{\eta} H_{\eta}\left(u_{\eta}-u_{\eta, s}\right) \cdot \xi d x=\omega_{\xi} \cdot\left(J_{\eta} \omega_{u}\right)-\omega_{\xi} \cdot\left(J_{\eta} \omega_{u}\right)=0 .
$$

\subsection{Estimates for transport and Navier-Stokes equations}

In all the sequel, $C$ denotes a positive constant. At this stage, we consider a given time interval $[0, T]$. The value to which $T$ must be restricted will be given later in this section.

Standard estimates for transport equations (15) and (16) show that $\rho_{\eta}$ and $H_{\eta}$ are bounded in $L^{\infty}\left(0, T ; L^{\infty}(\Omega)\right)$. More precisely, for all time $t \in[0, T]$,

$$
\rho_{\min }:=\min \left(\rho_{s}, \rho_{f}\right) \leq \rho_{\eta}(x, t) \leq \max \left(\rho_{s}, \rho_{f}\right) \quad H_{\eta}(x, t) \in\{0,1\} \quad \text { a.e. } x \in \Omega \text {. }
$$


Thus, up to extracting a subsequence, we can assume that

$$
\rho_{\eta} \rightarrow \rho \text { in } L^{\infty}\left(0, T, L^{\infty}(\Omega)\right) \text { weak }{ }^{*}
$$

and

$$
H_{\eta} \rightarrow H \text { in } L^{\infty}\left(0, T, L^{\infty}(\Omega)\right) \text { weak* }
$$

where $H$ and $\rho$ satisfy the bounds (18). We set $\Omega_{s}(t)=\{x \in \Omega, H(x, t)=1\}$. Concerning the Navier-Stokes equations, using $u_{\eta}$ as a test function in the weak formulation of (12) and using (15) we classically obtain:

$$
\frac{1}{2} \int_{\Omega} \frac{\partial\left(\rho_{\eta}\left|u_{\eta}\right|^{2}\right)}{\partial t} d x+2 \mu \int_{\Omega}\left|D\left(u_{\eta}\right)\right|^{2} d x+\frac{1}{\eta} \int_{\Omega} \rho_{\eta} H_{\eta}\left(u_{\eta}-u_{\eta, s}\right) \cdot u_{\eta} d x=\int_{\Omega} \rho_{\eta} g \cdot u_{\eta} d x .
$$

From Lemma 3.1, since $u_{\eta, s}$ is a rigid velocity field, we get

$$
\int_{\Omega} \rho_{\eta} H_{\eta}\left(u_{\eta}-u_{\eta, s}\right) \cdot u_{\eta} d x=\int_{\Omega} \rho_{\eta} H_{\eta}\left(u_{\eta}-u_{\eta, s}\right)^{2} d x
$$

Collecting terms we get, since from (18) $\sqrt{H_{\eta}}=H_{\eta}$,

$\frac{1}{2} \frac{d}{d t}\left\|\sqrt{\rho_{\eta}} u_{\eta}\right\|_{L^{2}(\Omega)}^{2}+\mu\left\|D\left(u_{\eta}\right)\right\|_{L^{2}(\Omega)}^{2}+\frac{1}{\eta}\left\|\sqrt{\rho_{\eta}} H_{\eta}\left(u_{\eta}-u_{\eta, s}\right)\right\|_{L^{2}(\Omega)}^{2} \leq\left\|\sqrt{\rho_{\eta}} u_{\eta}\right\|_{L^{2}(\Omega)}\|g\|_{L^{\infty}(Q)}\left\|\sqrt{\rho_{\eta} \|}\right\|_{L^{2}(Q)}$

which upon time integration on $[0, T]$ gives

$$
\begin{aligned}
\left\|\sqrt{\rho_{\eta}}(t) u_{\eta}(t)\right\|_{L^{2}(\Omega)}^{2} & +2 \mu\left\|D\left(u_{\eta}\right)\right\|_{L^{2}(Q)}^{2}+\frac{2}{\eta}\left\|\sqrt{\rho_{\eta}} H_{\eta}\left(u_{\eta}-u_{\eta, s}\right)\right\|_{L^{2}(Q)}^{2} \\
& \leq\left\|\sqrt{\rho_{\eta 0}} u_{\eta 0}\right\|_{L^{2}(\Omega)}^{2}+C \int_{0}^{T}\left\|\sqrt{\rho_{\eta}}(s) u_{\eta}(s)\right\|_{L^{2}(\Omega)} d s .
\end{aligned}
$$

Applying Gronwall Lemma, Poincaré inequality and bounds from (18) gives the following estimates :

$$
\begin{gathered}
u_{\eta} \text { bounded in } L^{2}\left(0, T, H_{0}^{1}(\Omega)\right), \\
\sqrt{\rho_{\eta}} u_{\eta} \text { and } u_{\eta} \text { bounded in } L^{\infty}\left(0, T, L^{2}(\Omega)\right), \\
\frac{1}{\sqrt{\eta}} \sqrt{\rho_{\eta}} H_{\eta}\left(u_{\eta}-u_{\eta, s}\right) \text { and } \frac{1}{\sqrt{\eta}} H_{\eta}\left(u_{\eta}-u_{\eta, s}\right) \text { bounded in } L^{2}\left(0, T, L^{2}(\Omega)\right) .
\end{gathered}
$$

Thus we can extract subsequences from $\rho_{\eta}, u_{\eta}$ and $H_{\eta}$, still denoted by $\rho_{\eta}, u_{\eta}$ and $H_{\eta}$, such that

$$
u_{\eta} \rightarrow u \text { in } L^{2}\left(0, T, H_{0}^{1}(\Omega)\right) \text { weak }
$$




$$
\begin{gathered}
\sqrt{\rho_{\eta}} u_{\eta} \rightarrow \chi \text { and } u_{\eta} \rightarrow u \text { in } L^{\infty}\left(0, T, L^{2}(\Omega)\right) \text { weak* } \\
\sqrt{\rho_{\eta}} H_{\eta} u_{\eta}-\sqrt{\rho}_{\eta} H_{\eta} u_{\eta, s} \rightarrow 0 \text { and } H_{\eta} u_{\eta}-H_{\eta} u_{\eta, s} \rightarrow 0 \text { in } L^{2}\left(0, T, L^{2}(\Omega)\right) \text { strong. }
\end{gathered}
$$

The identification of $\chi$ with $\sqrt{\rho} u$ results from strong convergence results proved by Lions and DiPerna on transport equations. [9] theorem II.4, (24) and incompressibility imply

$$
\rho_{\eta} \rightarrow \rho \text { in } C\left(0, T, L^{q}(\Omega)\right) \text { strong } \forall q \in[1,+\infty[
$$

with $\rho$ solution of

$$
\begin{cases}\rho_{t}+u \cdot \nabla \rho=0 & \text { on } \Omega \times] 0, T[, \\ \rho=\rho_{0} & \text { on } \Omega \times\{0\} .\end{cases}
$$

From this strong convergence we can pass to the limit in the product $\sqrt{\rho_{\eta}} u_{\eta}$ : given $v \in$ $L^{q}\left(0, T ; L^{r}(\Omega)\right)$ with $q>2$ and $r>\frac{6}{5}$, we write

$$
\int_{0}^{T} \int_{\Omega}\left(\sqrt{\rho_{\eta}} u_{\eta}-\sqrt{\rho} u\right) v d x d t=\int_{0}^{T} \int_{\Omega}\left(u_{\eta}-u\right) \sqrt{\rho} v d x d t+\int_{0}^{T} \int_{\Omega}\left(\sqrt{\rho}_{\eta}-\sqrt{\rho}\right) u_{\eta} v d x d t .
$$

From the injection of $H^{1}$ into $L^{6}$ in dimension less or equal to 3 the first integral converges toward 0 . For the second integral we use the strong convergence (following (27)) of $\sqrt{\rho_{\eta}}$ in $L^{s}$ for a $s$ such that $u_{\eta} v$ is in $L^{s^{\prime}}$ where $s^{\prime}$ is the conjugate exponent of $s$. Thus we have

$$
\sqrt{\rho_{\eta}} u_{\eta} \rightarrow \sqrt{\rho} u \text { in } L^{q}\left(0, T, L^{r}(\Omega)\right) \text { weak, for all } q<2, r<6 .
$$

\subsection{Setting $\mathrm{T}$ and passing to the limit in the rigid velocity}

This rigid velocity is defined by

$$
u_{\eta, s}(x, t)=u_{\eta, G}(t)+\omega_{\eta}(t) \times r_{\eta}(x, t),
$$

with

$$
u_{\eta, G}(t)=\frac{1}{M_{\eta}} \int_{\Omega} \rho_{\eta} u_{\eta} H_{\eta} d x \quad \text { and } \quad \omega_{\eta}(t)=J_{\eta}^{-1} \int_{\Omega} \rho_{\eta}\left(r_{\eta} \times u_{\eta}\right) H_{\eta} d x .
$$

First we note that $M_{\eta}=\int_{\Omega} \rho_{\eta} H_{\eta} d x$ is bounded from below independently of $\eta$ since $\rho_{\eta}$ is bounded from below and $\int_{\Omega} H_{\eta} d x=\left|\Omega_{s}^{0}\right|>0$ does not depend on $\eta$ or $t$. From the bounds on $\rho_{\eta}, H_{\eta}$ and $u_{\eta}$ it is straightforward to show that

$$
u_{\eta, G}(t) \text { bounded in } L^{\infty}(0, T) \text {. }
$$


Likewise, from the definition of $J_{\eta}$ we observe that for $a \in \mathbb{R}^{3} \backslash\{0\}$

$$
a^{T} J_{\eta} a \geq \min \left(\rho_{s}, \rho_{f}\right) \int_{\Omega_{s}(t)}\left|r_{\eta} \times a\right|^{2} d x>0 .
$$

Moreover, the initial solid is regular and transported by a rigid velocity. We thus know that there is a ball of radius $R>0$ centered on the center of gravity $x_{G \eta}$ included into $\Omega_{s}^{\eta}(t)$. Then the above estimates implies

$$
a^{T} J_{\eta} a \geq \min \left(\rho_{s}, \rho_{f}\right) \int_{B\left(x_{G \eta}, R\right)}\left|r_{\eta} \times a\right|^{2} d x=\min \left(\rho_{s}, \rho_{f}\right) \int_{B(0, R)}|x \times a|^{2} d x=C(R)|a|^{2}
$$

with $C(R)=\frac{2 R^{5} \pi}{15}>0$. Taking $a=J_{\eta}^{-\frac{1}{2}} b\left(J_{\eta}\right.$ is symmetric $)$ we get for all $b \in \mathbb{R}^{3} \backslash\{0\}$,

$$
b^{T} J_{\eta}^{-1} b=\left|J_{\eta}^{-\frac{1}{2}} b\right|^{2} \leq \frac{1}{C(R)}|b|^{2},
$$

which proves that each coefficient of $J_{\eta}^{-1}$ is bounded independently of $\eta$ and $t$. From the bounds on $u_{\eta}, H_{\eta}$ and $\rho_{\eta}$ this implies that

$$
\omega_{\eta}(t) \text { is bounded in } L^{\infty}(0, T) .
$$

In particular this implies that the solid velocity $u_{\eta, s}$ is bounded in $L^{\infty}$ by some constant $M$ independent of $\eta$ and time. We can now define the maximum time for which the convergence result will be proved. If we denote by $d_{0}$ the initial distance between solid and the boundary $\partial \Omega$ then choosing for instance $T=d_{0} / 2 M$ ensures that the body will not touch the boundary for $t \in[0, T]$. In all the sequel we will assume this value of $T$.

From the above estimates we can ensure that there exists $u_{G}(t)$ and $\omega(t)$ in $L^{\infty}(0, T)$ such that, up to the extraction of subsequences,

$$
u_{\eta, s} \rightarrow u_{s}:=u_{G}+\omega \times r \text { in } L^{\infty}\left(0, T, L^{\infty}(\Omega)\right) \text { weak* } .
$$

Now we point out that taking the gradient of the rigid velocity field $u_{\eta, s}$ gives

$$
\nabla u_{\eta, s}=\left(\begin{array}{ccc}
0 & -\omega_{\eta}^{3} & \omega_{\eta}^{2} \\
\omega_{\eta}^{3} & 0 & -\omega_{\eta}^{1} \\
-\omega_{\eta}^{2} & \omega_{\eta}^{1} & 0
\end{array}\right)
$$

so that the $\nabla u_{\eta, s}$ (and all subsequent space derivatives) is also bounded in $L^{\infty}\left(0, T ; L^{\infty}(\Omega)\right.$ ). In particular

$$
u_{\eta, s} \rightarrow u_{s} \text { in } L^{2}\left(0, T, W^{1, \infty}(\Omega)\right) \text { weak* }
$$


We now wish to prove that $u_{s}$ (or equivalently $u_{G}$ and $\omega$ ) has a similar structure as $u_{\eta, s}$, that is, to pass to the limit in the expression defining $u_{\eta, s}$. Using (29), the already mentioned compactness results of [9], applied to the transport equation on $H_{\eta}$ now gives

$$
H_{\eta} \rightarrow H \text { a.e. and in } C\left(0, T, L^{p}(\Omega)\right) \text { strong } \forall p \in[1,+\infty[
$$

with $H$ verifying

$$
\left\{\begin{array}{l}
H_{t}+u_{s} . \nabla H=0 \text { on } \mathbb{R}^{n} \times(0, T) \\
H=H_{0} \text { on } \mathbb{R}^{n} \times\{0\} .
\end{array}\right.
$$

Note that this Cauchy problem has been set in $\mathbb{R}^{n}$ because $u_{s}$ does not vanish on $\partial \Omega$, but $H$ vanishes outside $\Omega$. However we can prove by passing to the limit in (26) that $H u=H u_{s}$. Thus $\operatorname{div}(u H)=\operatorname{div}\left(u_{s} H\right)$ and $H$ verifies a transport equation with velocity field $u$ on $\Omega$ (note that no boundary conditions are needed on $\partial \Omega$ since $u$ is zero on the boundary).

This convergence gives us the strong convergence of $r_{\eta}$ in $\mathcal{C}\left(0, T, L^{p}(\Omega)\right), \forall p \geq 1$, and from $(27),(30)$ and (24) we can easily pass to the limit in the expression of $u_{\eta, G}$ and $\omega_{\eta}$ to get

$$
u_{G}(t)=\frac{\int_{\Omega} \rho u H d x}{\int_{\Omega} \rho H d x} \quad \text { and } \quad \omega(t)=\left(\int_{\Omega} \rho\left(r^{2} \mathbb{I}-r \otimes r\right) H d x\right)^{-1} \int_{\Omega} \rho(r \times u) H d x .
$$

\subsection{Strong convergence of $u_{\eta}$}

The remaining part of the proof is more technical since we aim to prove the strong convergence of at least a subsequence of $u_{\eta}$ in order to be able to pass to the limit in the inertial term of Navier-Stokes equations. Classically, this is obtained thanks to a Fourier transform in time which provides an estimate on some fractional time derivative of $u_{\eta}$ which brings compactness $[16,23]$. Here these technics can not be used since the solid is moving. We instead rely on tools developed in $[20]$.

Thereafter we will use, for $\sigma>0$ and $r \in[1 / 2,1]$, the following notations

- $\Omega_{s, \sigma}(t)=\left\{x \in \Omega, d\left(x, \Omega_{s}(t)\right)<\sigma\right\}$,

- $\mathcal{V}^{0}=\left\{v \in L^{2}(\Omega), \operatorname{div} v=0, v \cdot n=0\right.$ on $\left.\partial \Omega\right\}$,

- $\mathcal{V}^{r}=\left\{v \in H^{r}(\Omega), \operatorname{div} v=0, v=0\right.$ on $\left.\partial \Omega\right\}$,

- $\mathcal{K}_{\sigma}^{r}(t)=\left\{v(t) \in \mathcal{V}^{r}, D(v(t))=0\right.$ in $\left.\mathcal{D}^{\prime}\left(\Omega_{s, \sigma}(t)\right)\right\}$ (which is a closed subset of $\left.\mathcal{V}^{r}\right)$, 
- $P_{\sigma}^{r}(t)$ the orthogonal projection in the sense of the $H^{r}$ norm of $\mathcal{V}^{r}$ on $\mathcal{K}_{\sigma}^{r}(t)$.

To prove the strong convergence of a subsequence of $u_{\eta}$ in $L^{2}(Q)$ we write

$$
\int_{0}^{T} \int_{\Omega}\left|u_{\eta}-u\right|^{2} d x d t \leq \frac{1}{\rho_{\min }}\left(\int_{0}^{T} \int_{\Omega}\left|\rho\left(u_{\eta}^{2}-u^{2}\right)\right| d x d t+\int_{0}^{T} \int_{\Omega}\left|2 \rho u \cdot\left(u-u_{\eta}\right)\right| d x d t\right) .
$$

From (25) the second integral on the right side converges to 0 , thus

$$
\int_{0}^{T} \int_{\Omega}\left|u_{\eta}-u\right|^{2} d x d t \leq \frac{1}{\rho_{\min }}\left(\int_{0}^{T} \int_{\Omega}\left|\rho_{\eta} u_{\eta}^{2}-\rho u^{2}\right| d x d t+\int_{0}^{T} \int_{\Omega}\left|\left(\rho_{\eta}-\rho\right) u_{\eta}^{2}\right| d x d t\right)+l_{\eta},
$$

where $l_{\eta} \rightarrow 0$ when $\eta \rightarrow 0$. Moreover, by (21) and (27) the second integral on the right hand side converges to 0 , and

$$
\begin{aligned}
\int_{0}^{T} \int_{\Omega}\left|u_{\eta}-u\right|^{2} d x d t & \leq \frac{1}{\rho_{\min }}\left(\int_{0}^{T} \int_{\Omega}\left|\rho_{\eta} u_{\eta} \cdot P_{\sigma}^{r}\left(u_{\eta}\right)-\rho u \cdot P_{\sigma}^{r}(u)\right| d x d t\right. \\
& \left.+\int_{0}^{T} \int_{\Omega}\left|\rho_{\eta} u_{\eta} \cdot\left(u_{\eta}-P_{\sigma}^{r}\left(u_{\eta}\right)\right)\right| d x d t+\int_{0}^{T} \int_{\Omega}\left|\rho u \cdot\left(P_{\sigma}^{r}(u)-u\right)\right| d x d t+l_{\eta}\right) \\
& \leq \frac{1}{\rho_{\min }}\left(\left\|\rho_{\eta} u_{\eta} \cdot P_{\sigma}^{r}\left(u_{\eta}\right)-\rho u \cdot P_{\sigma}^{r}(u)\right\|_{L^{1}(Q)}\right. \\
& +\left\|\rho_{\eta}\right\|_{L^{\infty}(Q)}\left\|u_{\eta}\right\|_{L^{2}(Q)}\left\|P_{\sigma}^{r}\left(u_{\eta}\right)-u_{\eta}\right\|_{L^{2}(Q)} \\
& +\|\rho\|_{L^{\infty}(Q)}\|u\|_{L^{2}(Q)}\left\|P_{\sigma}^{r}(u)-u\right\|_{L^{2}(Q)}+l_{\eta}
\end{aligned}
$$

Finally, as $\rho_{\eta}$ is bounded in $L^{\infty}\left(0, T, L^{\infty}(\Omega)\right)$ and using (21), we get

$$
\begin{aligned}
\int_{0}^{T} \int_{\Omega}\left|u_{\eta}-u\right|^{2} d x d t & \leq \frac{1}{\rho_{\min }}\left(\left\|\rho_{\eta} u_{\eta} \cdot P_{\sigma}^{r}\left(u_{\eta}\right)-\rho u \cdot P_{\sigma}^{r}(u)\right\|_{L^{1}(Q)}\right. \\
& +C\left\|P_{\sigma}^{r}\left(u_{\eta}\right)-u_{\eta}\right\|_{L^{2}(Q)} \\
& +C\left\|P_{\sigma}^{r}(u)-u\right\|_{L^{2}(Q)}+l_{\eta}
\end{aligned}
$$

This decomposition shows that the sought convergence essentially amounts to prove that (up to the extraction of subsequences)

$$
\begin{array}{r}
\lim _{\sigma \rightarrow 0}\left\|P_{\sigma}^{r}(u)-u\right\|_{L^{2}(Q)}=0 \\
\lim _{\sigma \rightarrow 0} \lim _{\eta \rightarrow 0}\left\|P_{\sigma}^{r}\left(u_{\eta}\right)-u_{\eta}\right\|_{L^{2}(Q)}=0 \\
\lim _{\sigma \rightarrow 0} \lim _{\eta \rightarrow 0}\left\|\rho_{\eta} u_{\eta} \cdot P_{\sigma}^{r}\left(u_{\eta}\right)-\rho u \cdot P_{\sigma}^{r}(u)\right\|_{L^{1}(Q)}=0
\end{array}
$$

To prove (32-34) we will make use of some Lemma that we state now.

Lemma 3.2. Let $\left(f_{n}\right)$ be a sequence of functions bounded in $L^{p}(0, T)$ for some $p>2$ and converging to 0 almost everywhere on $[0, T]$. Then $\left(f_{n}\right)$ converges strongly to 0 in $L^{2}(0, T)$. 
Proof. Let $\varepsilon>0$. From Egorov theorem ([5], p.75), the almost everywhere convergence implies that there exists $A_{\varepsilon} \subset[0, T]$ such that

$$
\left\{\begin{array}{l}
\left|[0, T] \backslash A_{\varepsilon}\right|<\varepsilon \\
f_{n} \rightarrow 0 \text { uniformly on } A_{\varepsilon}
\end{array}\right.
$$

which means

$$
\exists N \in \mathbb{N}, \forall n \geq N, \forall t \in A_{\varepsilon},\left|f_{n}(t)\right|^{2}<\varepsilon
$$

Therefore

$$
\int_{A_{\varepsilon}}\left(f_{n}(t)\right)^{2} d t \leq \varepsilon\left|A_{\varepsilon}\right| \leq \varepsilon T .
$$

Since, from the bound in the $L^{p}$ norm

$$
\int_{[0, T] \backslash A_{\varepsilon}}\left(f_{n}(t)\right)^{2} d t \leq\left(\int_{[0, T] \backslash A_{\varepsilon}} 1^{q} d t\right)^{2 / q}\left(\int_{[0, T]}\left(f_{n}(t)\right)^{p} d t\right)^{2 / p} \leq \varepsilon^{2 / q} C
$$

with $\frac{1}{q}+\frac{1}{p}=\frac{1}{2}$, we get

$$
\int_{0}^{T}\left(f_{n}(t)\right)^{2} d t \leq \varepsilon T+\varepsilon^{2 / q} C
$$

which proves the $L^{2}$ convergence.

Lemma 3.3. Let $u(t) \in H^{1}\left(\Omega_{s, \sigma}(t)\right)$ such that $u_{\mid \partial \Omega_{s, \sigma}(t)}(t)=g(t)$ and $(w(t), p(t)) \in H^{1}\left(\Omega \backslash \overline{\Omega_{s, \sigma}}(t)\right) \times$ $L^{2}\left(\Omega \backslash \overline{\Omega_{s, \sigma}}(t)\right)$ solution of the Stokes problem

$$
\begin{cases}-\Delta w(t)+\nabla p(t)=0 & \text { on } \Omega \backslash \overline{\Omega_{s, \sigma}}(t), \\ \operatorname{div} w(t)=0 & \text { on } \Omega \backslash \overline{\Omega_{s, \sigma}}(t), \\ w(t)=g(t) & \text { on } \partial \Omega_{s, \sigma}(t), \\ w(t)=0 & \text { on } \partial \Omega .\end{cases}
$$

Then there exists $\sigma_{0}>0$ and $C>0$, such that for all $\sigma<\sigma_{0}$, we have the following estimate:

$$
\|w(t)\|_{L^{2}\left(\Omega \backslash \overline{\left.\Omega_{s, \sigma}(t)\right)}\right.}^{2} \leq C\|u(t)\|_{L^{2}\left(\Omega_{s, \sigma}(t)\right)}\|\nabla u(t)\|_{L^{2}\left(\Omega_{s, \sigma}(t)\right)}
$$

The proof of this result is postponed to the appendix.

Lemma 3.4. The limits $H, u$ and $u_{s}$ defined in (30), (24) and (29) verify

$$
H u=H u_{s} .
$$

Proof. First we claim that

$$
H_{\eta} u_{\eta} \rightarrow H u \text { in } L^{q}\left(0, T, L^{r}(\Omega)\right) \text { weak, with } q<2 \text { and } r<6
$$


Indeed, let us introduce $v \in L^{q}\left(0, T, L^{r}(\Omega)\right)$ with $q>2$ and $r>\frac{6}{5}$. We have

$$
\int_{0}^{T} \int_{\Omega}\left(H_{\eta} u_{\eta}-H u\right) \cdot v d x d t=\int_{0}^{T} \int_{\Omega} H\left(u_{\eta}-u\right) \cdot v d x d t+\int_{0}^{T} \int_{\Omega}\left(H_{\eta}-H\right) u_{\eta} \cdot v d x d t
$$

From the injection of $H^{1}$ into $L^{6}$ in dimension less or equal to 3 and (24) we get

$$
u_{\eta} \rightarrow u \text { in } L^{2}\left(0, T, L^{6}(\Omega)\right) \text { weak, }
$$

and, since $H$ is bounded in $L^{\infty}(Q)$,

$$
\lim _{\eta \rightarrow 0} \int_{0}^{T} \int_{\Omega} H\left(u_{\eta}-u\right) \cdot v d x d t=0 .
$$

Moreover from (30) we easily get

$$
\lim _{\eta \rightarrow 0} \int_{0}^{T} \int_{\Omega}\left(H_{\eta}-H\right) u_{\eta} \cdot v d x d t=0 .
$$

We next show that

$$
H_{\eta} u_{\eta, s} \rightarrow H u_{s} \text { in } L^{p}\left(0, T, L^{p}(\Omega)\right) \text { weak, } \forall p \in[1,+\infty[.
$$

Let $v \in L^{p}\left(0, T, L^{p}(\Omega)\right)$ with $p>1$. We write

$$
\int_{0}^{T} \int_{\Omega}\left(H_{\eta} u_{\eta, s}-H u_{s}\right) \cdot v d x d t=\int_{0}^{T} \int_{\Omega} H\left(u_{\eta, s}-u_{s}\right) \cdot v d x d t+\int_{0}^{T} \int_{\Omega}\left(H_{\eta}-H\right) u_{\eta, s} \cdot v d x d t .
$$

As $H$ is bounded in $L^{\infty}(Q)$, with (29) we get:

$$
\lim _{\eta \rightarrow 0} \int_{0}^{T} \int_{\Omega} H\left(u_{\eta, s}-u_{s}\right) \cdot v d x d t=0
$$

In addition, from (30) we get

$$
\lim _{\eta \rightarrow 0} \int_{0}^{T} \int_{\Omega}\left(H_{\eta}-H\right) u_{\eta, s} \cdot v d x d t=0 .
$$

By (36) and (37) we thus deduce

$$
H_{\eta} u_{\eta}-H_{\eta} u_{\eta, s} \rightarrow H u-H u_{s} \text { in } L^{q}\left(0, T, L^{r}(\Omega)\right) \text { weak, with } q<2 \text { and } r<6
$$

Finally we recall that

$$
H_{\eta} u_{\eta}-H_{\eta} u_{\eta, s} \rightarrow 0 \text { in } L^{2}(Q) \text { strong, }
$$

and the desired result follows. The result is finally obtained by identifying the limits in (38) et (39). 


\section{Lemma 3.5.}

$$
\forall \sigma>0, \quad \exists \eta_{0}>0, \quad \forall \eta<\eta_{0}, \quad \forall t \in[0, T], \quad \Omega_{s}^{\eta}(t) \subset \Omega_{s, \sigma}(t) \text { and } \Omega_{s}(t) \subset \Omega_{s, \sigma}^{\eta}(t)
$$

Proof. From (30) with $p=1$ we have

$$
\forall \varepsilon>0, \quad \exists \eta_{0}>0, \quad \forall \eta<\eta_{0}, \quad \forall t \in[0, T], \quad \int_{\Omega}\left|H_{\eta}(x, t)-H(x, t)\right| d x<\varepsilon,
$$

which means

$$
\forall \varepsilon>0, \quad \exists \eta_{0}>0, \quad \forall \eta<\eta_{0}, \quad \forall t \in[0, T], \quad\left|\Omega_{s}^{\eta}(t) \backslash \Omega_{s}(t)\right|+\left|\Omega_{s}(t) \backslash \Omega_{s}^{\eta}(t)\right|<\varepsilon
$$

By contradiction we suppose that we can find $\sigma_{0}>0$ such as $\forall \eta_{0}>0$, there exists $\eta<\eta_{0}$ and $t \in[0, T]$ for which at least one of the inclusions of (40) is false. Assume the first inclusion is false.This means that we can find $x_{\eta}(t) \in \Omega_{s}^{\eta}(t)$ such as $d\left(x_{\eta}(t), \Omega_{s}(t)\right)>\sigma_{0} . \Omega_{s}^{\eta}(t)$ is a rigid deformation of $\Omega_{s}^{\eta}(0)$ so its boundary is $\mathcal{C}^{2}$. Thus, there exists a sufficiently small $\rho$ independent of $\eta$, such as for each point of $\Omega_{s}^{\eta}(t)$ there exists a ball of radius $\rho>0$ containing this point and included in $\Omega_{s}^{\eta}(t)$. Then there exists also a ball of radius $\bar{\rho}:=\min \left(\rho, \sigma_{0} / 3\right)$ containing the point and included in $\Omega_{s}^{\eta}(t)$. This latter ball is included in $\Omega_{s}^{\eta}(t) \backslash \Omega_{s}(t)$. Indeed it contains a point at distance more than $\sigma_{0}$ from $\Omega_{s}(t)$ and its diameter is less than $2 \sigma_{0} / 3$. We thus obtained that

$$
\exists \sigma_{0}, \forall \eta_{0}>0, \exists \eta>0, \exists t \in[0, T],\left|\Omega_{s}^{\eta}(t) \backslash \Omega_{s}(t)\right|>\pi \bar{\rho}^{2}
$$

with $\bar{\rho}$ independent of $\eta$ and $t$. This contradicts (42). A similar argument shows that the second inclusion cannot hold either.

A key point in the convergence is the following result.

\section{Lemma 3.6.}

$$
\lim _{\sigma \rightarrow 0}\left\|P_{\sigma}^{r}(u)-u\right\|_{L^{2}\left(0, T, \mathcal{V}^{r}\right)}=0, \forall r \in[1 / 2,1[
$$

The proof of this result is postponed to the appendix.

The next lemma, which is also proved in the appendix, is essentially a rephrasing of the previous one with $u_{\eta}$ instead of $u$. The difference is that we do not have anymore $u_{\eta}-u_{\eta, s}=0$ ins $\Omega_{s}^{\eta}(t)$, but we do have an estimate on it, from (23), which allows to pass to the limit as $\eta$ goes to 0.

\section{Lemma 3.7.}

$$
\left.\lim _{\sigma \rightarrow 0} \lim _{\eta \rightarrow 0}\left\|P_{\sigma}^{r}\left(u_{\eta}\right)-u_{\eta}\right\|_{L^{2}\left(0, T, \mathcal{V}^{r}\right)}=0, \forall r \in\right] 1 / 2,1[
$$




\section{Lemma 3.8.}

$$
\left.\lim _{\sigma \rightarrow 0} \lim _{\eta \rightarrow 0}\left\|\rho_{\eta} u_{\eta} \cdot P_{\sigma}^{r}\left(u_{\eta}\right)-\rho u \cdot P_{\sigma}^{r}(u)\right\|_{L^{1}([0, T] \times \Omega)}=0, \forall r \in\right] 1 / 2,1[.
$$

Proof. Let $r \in] 1 / 2,1\left[\right.$ and $\sigma>0$. From Lemma 3.5 there exists $\eta_{0}>0$ such that $\forall \eta<\eta_{0}$,

$$
\Omega_{s}^{\eta}(t) \subset \Omega_{s, \sigma / 3}(t), \forall t \in[0, T]
$$

Let $\eta<\eta_{0}$. Arguing as in [20] we split $[0, T]$ in $N_{T}$ subintervals $I_{k}=[(k-1) \tau, k \tau], \tau=T / N_{T}$, $k=1, . ., N_{T}$. We choose $N_{T}$ large enough (depending on $\sigma$ ) such that

$$
\Omega_{s, \sigma / 2}(k \tau) \subset \Omega_{s, \sigma}(t) \text { and } \Omega_{s, \sigma / 3}(t) \subset \Omega_{s, \sigma / 2}(k \tau), \quad \forall t \in I_{k}, \forall k=1, . ., N_{T} .
$$

This is possible because $\Omega_{s, \sigma}(t)$ is moving with a rigid velocity field, with $L^{2}$ regularity in time : the flow $X(t, x)$ generated by this velocity field is thus continuous in time. Let $x \in \Omega_{s, \sigma / 2}(k \tau)$. There exits $y \in \Omega_{s}(k \tau)$ such that $|y-x| \leq \sigma / 2$. But $y=X(k \tau, z)$ for some $z \in \Omega_{s}(0)$ and therefore $|y-X(t, z)| \leq \sigma / 2$ for $t \in I_{k}$ if $\tau$ is small enough. Therefore $|x-X(t, z)| \leq \sigma$. This proves the first inclusion in (46). The second inclusion is proved by a similar continuity argument. On each subinterval $I_{k}, k=1, . ., N_{T}$, we consider the momentum equation

$$
\rho_{\eta} \frac{\partial u_{\eta}}{\partial t}+\rho_{\eta}\left(u_{\eta} \cdot \nabla\right) u_{\eta}-2 \mu \operatorname{div}\left(D\left(u_{\eta}\right)\right)+\nabla p_{\eta}+\frac{1}{\eta} \rho_{\eta} H_{\eta}\left(u_{\eta}-u_{\eta, s}\right)-\rho_{\eta} g=0 .
$$

Let us consider a test function $\xi$ vanishing outside $I_{k}$ and such that $\xi(., t) \in \mathcal{K}_{\sigma / 2}^{1}(k \tau)$ for $t \in I_{k}$. Since $\xi(., t)$ is rigid on $\Omega_{s, \sigma / 2}(k \tau) \supset \Omega_{s, \sigma / 3}(t) \supset \Omega_{s}^{\eta}(t)$, Lemma 3.1 yields

$$
\int_{I_{k}} \int_{\Omega}\left[\rho_{\eta} u_{\eta} \cdot \xi_{t}+\left(\rho_{\eta} u_{\eta} \otimes u_{\eta}-2 \mu D\left(u_{\eta}\right)\right): D(\xi)+\rho_{\eta} g \cdot \xi\right] d x d t=0 .
$$

From bounds given by (18), (21) and (22) we derive the following estimates:

$$
\begin{aligned}
\left|\int_{I_{k}} \int_{\Omega} D\left(u_{\eta}\right): D(\xi) d x d t\right| & \leq\left\|D\left(u_{\eta}\right)\right\|_{L^{2}\left(I_{k}, L^{2}(\Omega)\right)}\|D(\xi)\|_{L^{2}\left(I_{k}, L^{2}(\Omega)\right)} \\
& \leq C\|\xi\|_{L^{2}\left(I_{k}, H_{0}^{1}(\Omega)\right)} \leq C\|\xi\|_{L^{2}\left(I_{k}, \mathcal{K}_{\sigma / 2}^{1}(k \tau)\right)} \\
& \leq C\|\xi\|_{L^{4}\left(I_{k}, \mathcal{K}_{\sigma / 2}^{1}(k \tau)\right)},
\end{aligned}
$$




$$
\begin{aligned}
\left|\int_{I_{k}} \int_{\Omega}\left(\rho_{\eta} u_{\eta} \otimes u_{\eta}\right): D(\xi) d x d t\right| & \leq \int_{I_{k}}\left\|\rho_{\eta}\right\|_{L^{\infty}(\Omega)}\left\|u_{\eta} \otimes u_{\eta}\right\|_{L^{2}(\Omega)}\|D(\xi)\|_{L^{2}(\Omega)} d t \\
& \leq\left\|\rho_{\eta}\right\|_{L^{\infty}\left(I_{k}, L^{\infty}(\Omega)\right)} \int_{I_{k}}\left\|u_{\eta}\right\|_{L^{4}(\Omega)}^{2}\|\xi\|_{H_{0}^{1}(\Omega)} d t \\
& \leq C \int_{I_{k}}\left\|u_{\eta}\right\|_{L^{2}(\Omega)}^{\frac{1}{2}}\left\|\nabla u_{\eta}\right\|_{L^{2}(\Omega)}^{\frac{3}{2}}\|\xi\|_{H_{0}^{1}(\Omega)} d t \\
& \leq C\left\|u_{\eta}\right\|_{L^{\infty}\left(I_{k}, L^{2}(\Omega)\right)}^{\frac{1}{2}} \int_{I_{k}}\left\|\nabla u_{\eta}\right\|_{L^{2}(\Omega)}^{\frac{3}{2}}\|\xi\|_{H_{0}^{1}(\Omega)} d t \\
& \leq C\left\|\nabla u_{\eta}\right\|_{L^{2}\left(I_{k}, L^{2}(\Omega)\right)}^{\frac{3}{2}}\|\xi\|_{L^{4}\left(I_{k}, H_{0}^{1}(\Omega)\right)} \\
& \leq C\|\xi\|_{L^{4}\left(I_{k}, H_{0}^{1}(\Omega)\right)} \leq C\|\xi\|_{L^{4}\left(I_{k}, \mathcal{K}_{\sigma / 2}^{1}(k \tau)\right)},
\end{aligned}
$$

and

$$
\left|\int_{I_{k}} \int_{\Omega} \rho_{\eta} g \cdot \xi d x d t\right| \leq\left\|\rho_{\eta}\right\|_{L^{\infty}\left(I_{k}, L^{\infty}(\Omega)\right)}\|\xi\|_{L^{4}\left(I_{k}, \mathcal{K}_{\sigma / 2}^{1}(k \tau)\right)} \leq C\|\xi\|_{L^{4}\left(I_{k}, \mathcal{K}_{\sigma / 2}^{1}(k \tau)\right)} .
$$

Collecting terms we get

$$
\left|\int_{I_{k}} \int_{\Omega} \rho_{\eta} u_{\eta} \cdot \xi_{t} d x d t\right| \leq C\|\xi\|_{L^{4}\left(I_{k}, \mathcal{K}_{\sigma / 2}^{1}(k \tau)\right)} .
$$

As $\xi(., t) \in \mathcal{K}_{\sigma / 2}^{1}(k \tau), \xi_{t}(., t) \in \mathcal{K}_{\sigma / 2}^{1}(k \tau) \subset \mathcal{K}_{\sigma / 2}^{0}(k \tau)$ and we have

$$
\begin{aligned}
\left|\left\langle\rho_{\eta} u_{\eta}, \xi_{t}\right\rangle\right| & =\left|\left\langle\rho_{\eta} u_{\eta}, P_{\sigma / 2}^{0}(k \tau) \xi_{t}\right\rangle\right| \\
& =\left|\left\langle P_{\sigma / 2}^{0}(k \tau)\left(\rho_{\eta} u_{\eta}\right), \xi_{t}\right\rangle\right| \\
& =\left|\left\langle\frac{d}{d t} P_{\sigma / 2}^{0}(k \tau)\left(\rho_{\eta} u_{\eta}\right), \xi\right\rangle\right| .
\end{aligned}
$$

Therefore

$$
\left|\int_{I_{k}} \int_{\Omega} \frac{d}{d t} P_{\sigma / 2}^{0}(k \tau)\left(\rho_{\eta} u_{\eta}\right) \cdot \xi d x d t\right| \leq C\|\xi\|_{L^{4}\left(I_{k}, \mathcal{K}_{\sigma / 2}^{1}(k \tau)\right)}
$$

which means that

$$
\frac{d}{d t} P_{\sigma / 2}^{0}(k \tau)\left(\rho_{\eta} u_{\eta}\right) \text { bounded in } L^{\frac{4}{3}}\left(I_{k},\left(\mathcal{K}_{\sigma / 2}^{1}(k \tau)\right)^{*}\right)
$$

Moreover $\rho_{\eta} u_{\eta}$ is bounded in $L^{2}\left(I_{k}, L^{2}(\Omega)\right)$,

$$
P_{\sigma / 2}^{0}(k \tau)\left(\rho_{\eta} u_{\eta}\right) \text { is bounded in } L^{2}\left(I_{k}, \mathcal{K}_{\sigma / 2}^{0}(k \tau)\right)
$$

Since $\mathcal{K}_{\sigma / 2}^{0}(k \tau) \subset\left(\mathcal{K}_{\sigma / 2}^{r}(k \tau)\right)^{*}$ compactly for $r>0$, and $\left(\mathcal{K}_{\sigma / 2}^{r}(k \tau)\right)^{*} \subset\left(\mathcal{K}_{\sigma / 2}^{1}(k \tau)\right)^{*}$ continuously for $r<1$, by the Aubin-Simon Lemma (see e.g. [4], p. 98) with (47) and (48), we obtain the relative compactness of the sequence $\left(P_{\sigma / 2}^{0}(k \tau)\left(\rho_{\eta} u_{\eta}\right)\right)$ in $L^{2}\left(I_{k},\left(\mathcal{K}_{\sigma / 2}^{r}(k \tau)\right)^{*}\right)$ for all $\left.r \in\right] 1 / 2,1[$. From (24) we deduce

$$
\left.\lim _{\eta \rightarrow 0} P_{\sigma / 2}^{0}(k \tau)\left(\rho_{\eta} u_{\eta}\right)=P_{\sigma / 2}^{0}(k \tau) \rho u \text { in } L^{2}\left(I_{k},\left(\mathcal{K}_{\sigma / 2}^{r}(k \tau)\right)^{*}\right) \text { strong, } \forall r \in\right] 1 / 2,1[.
$$


Since from (46), we have

$$
\left.P_{\sigma / 2}^{0}(k \tau) P_{\sigma}^{r}(t)=P_{\sigma}^{r}(t) \forall t \in I_{k} \forall r \in\right] 1 / 2,1[
$$

and we can write

$$
\begin{aligned}
\int_{I_{k}}\left\langle\rho_{\eta} u_{\eta}, P_{\sigma}^{r}(t)\left(u_{\eta}\right)\right\rangle_{L^{2}(\Omega)} d t & =\int_{I_{k}}\left\langle\rho_{\eta} u_{\eta}, P_{\sigma / 2}^{0}(k \tau) P_{\sigma}^{r}(t) u_{\eta}\right\rangle_{L^{2}(\Omega)} d t \\
& =\int_{I_{k}}\left\langle P_{\sigma / 2}^{0}(k \tau)\left(\rho_{\eta} u_{\eta}\right), P_{\sigma}^{r}(t) u_{\eta}\right\rangle_{L^{2}(\Omega)} d t \\
& =\int_{I_{k}}\left\langle P_{\sigma / 2}^{0}(k \tau)\left(\rho_{\eta} u_{\eta}\right), P_{\sigma}^{r}(t) u_{\eta}\right\rangle_{\left(\mathcal{K}_{\sigma / 2}^{r}\right)^{*}, \mathcal{K}_{\sigma / 2}^{r}} d t
\end{aligned}
$$

The sequence $\left(u_{\eta}\right)$ is bounded in $L^{2}\left(0, T, \mathcal{V}^{r}\right)$ for all $\left.r \in\right] 1 / 2,1\left[\right.$, therefore $\left(P_{\sigma}^{r}(t) u_{\eta}\right)$ is bounded in $L^{2}\left(0, T, \mathcal{K}_{\sigma / 2}^{r}\right)$ for all $\left.r \in\right] 1 / 2,1[$.

Therefore there exists a subsequence of $P_{\sigma}^{r}(t) u_{\eta}$ still denoted $P_{\sigma}^{r}(t) u_{\eta}$ such that

$$
P_{\sigma}^{r}(t) u_{\eta} \rightarrow P_{\sigma}^{r}(t) u \text { in } L^{2}\left(0, T, \mathcal{K}_{\sigma / 2}^{r}\right) \text { weak. }
$$

Passing to the limit in $\eta$ yields

$$
\begin{aligned}
\lim _{\eta \rightarrow 0} \int_{I_{k}}\left\langle\rho_{\eta} u_{\eta}, P_{\sigma}^{r}(t)\left(u_{\eta}\right)\right\rangle_{L^{2}(\Omega)} d t & =\int_{I_{k}}\left\langle P_{\sigma / 2}^{0}(k \tau) \rho u, P_{\sigma}^{r}(t) u\right\rangle_{L^{2}(\Omega)} d t \\
& =\int_{I_{k}}\left\langle\rho u, P_{\sigma / 2}^{0}(k \tau) P_{\sigma}^{r}(t) u\right\rangle_{L^{2}(\Omega)} d t \\
& =\int_{I_{k}}\left\langle\rho u, P_{\sigma}^{r}(t) u\right\rangle_{L^{2}(\Omega)} d t .
\end{aligned}
$$

Summing over $k=1, . ., N_{T}$, we finally obtain

$$
\lim _{\eta \rightarrow 0}\left\|\rho_{\eta} u_{\eta} \cdot P_{\sigma}^{r}\left(u_{\eta}\right)-\rho u \cdot P_{\sigma}^{r}(u)\right\|_{L^{1}(Q)}=0
$$

which implies

$$
\lim _{\sigma \rightarrow 0} \lim _{\eta \rightarrow 0}\left\|\rho_{\eta} u_{\eta} \cdot P_{\sigma}^{r}\left(u_{\eta}\right)-\rho u \cdot P_{\sigma}^{r}(u)\right\|_{L^{1}(Q)}=0 .
$$

We can now conclude to the strong convergence of $u_{\eta}$.

Let $\varepsilon>0$. From Lemma 3.6,

$$
\exists \sigma_{0}>0, \forall \sigma<\sigma_{0},\left\|P_{\sigma}^{r}(u)-u\right\|_{L^{2}(Q)}<\varepsilon .
$$


From Lemma 3.7,

$$
\exists \sigma_{0}>0, \forall \sigma<\sigma_{0}, \exists \eta_{0}>0, \forall \eta<\eta_{0},\left\|P_{\sigma}^{r}\left(u_{\eta}\right)-u_{\eta}\right\|_{L^{2}(Q)}<\varepsilon
$$

and by Lemma 3.8 ,

$$
\exists \sigma_{0}>0, \forall \sigma<\sigma_{0}, \exists \eta_{0}>0, \forall \eta<\eta_{0},\left\|\rho_{\eta} u_{\eta} P_{\sigma}^{r}\left(u_{\eta}\right)-\rho u P_{\sigma}^{r}(u)\right\|_{L^{1}(Q)}<\varepsilon
$$

We therefore get from (31) (up to the extraction of a subsequence)

$$
\exists \eta_{0}>0, \forall \eta<\eta_{0}, \quad \int_{0}^{T} \int_{\Omega}\left|u_{\eta}-u\right|^{2} d x d t<C \varepsilon
$$

which means that (still up to a subsequence)

$$
u_{\eta} \rightarrow u \text { in } L^{2}(Q) \text { strong. }
$$

Classically, we also obtain from (19)

$$
\rho_{\eta} u_{\eta} \rightarrow \rho u \text { in } L^{2}(Q) \text { weak. }
$$

\subsection{Passing to the limit}

Let us now prove that as $\eta$ goes to zero, a subsequence of $\left(u_{\eta}, \rho_{\eta}\right)$ converges toward $(u, \rho)$ solution of the weak formulation (11). Indeed : We have proved that $\rho_{\eta} \rightarrow \rho$ in $L^{\infty}\left(0, T, L^{\infty}(\Omega)\right)$ weak * . Therefore

$$
\rho \in L^{\infty}(Q)
$$

We have proved that $u_{\eta} \rightarrow u$ in $L^{2}(0, T, V)$ weak, that $\sqrt{\rho_{\eta}} u_{\eta}$ is bounded in $L^{\infty}\left(0, T, L^{2}(\Omega)\right)$ and $\rho_{\eta}$ bounded from above and below in $L^{\infty}\left(0, T, L^{\infty}(\Omega)\right)$. This implies that $u_{\eta}$ bounded in $L^{\infty}\left(0, T, L^{2}(\Omega)\right)$, thus its weak limit belongs to $L^{\infty}\left(0, T, L^{2}(\Omega)\right) \cap L^{2}(0, T, V)$ :

$$
u \in L^{\infty}\left(0, T, L^{2}(\Omega)\right) \cap L^{2}(0, T, V) .
$$

From Lemma 3.4, we have $H u=H u_{s}=H\left(u_{G}+\omega \times r\right)$ where $H$ is the characteristic function of $\Omega_{s}(t)$. Thus

$$
u(t) \in \mathcal{K}(t)
$$

Using compactness results of DiPerna-Lions we already obtained that $\rho$ and $H$ are solutions of transport equations with $u$ and $u_{s}$ as velocities. For $H$ this means that for all $\psi \in \mathcal{C}^{1}(Q)$ with 
$\psi(T)=0$,

$$
\int_{0}^{T} \int_{\Omega} H \frac{\partial \psi}{\partial t}+H u_{s} \cdot \nabla \psi d x d t+\int_{\Omega} H^{0} \psi(0) d x=0
$$

As from Lemma 3.4, $H u_{s}=H u, H$ is also solution of

$$
\int_{0}^{T} \int_{\Omega} H \frac{\partial \psi}{\partial t}+H u \cdot \nabla \psi d x d t+\int_{\Omega} H^{0} \psi(0) d x=0 .
$$

In other terms $H$, like $\rho$ satisfies a transport equation with velocity $u$.

Let us finally check that $u$ satisfies the momentum equation.

Let $\sigma>0$. If $\xi_{\sigma} \in H^{1}(Q) \cap L^{2}\left(0, T ; \mathcal{K}_{\sigma}^{1}(t)\right)$, from (12) and (15) we get

$\int_{\Omega}\left[\frac{\partial\left(\rho_{\eta} u_{\eta}\right)}{\partial t}+\operatorname{div}\left(\rho_{\eta} u_{\eta} \otimes u_{\eta}\right)-2 \mu \operatorname{div}\left(D\left(u_{\eta}\right)\right)+\nabla p_{\eta}+\frac{1}{\eta} H_{\eta} \rho_{\eta}\left(u_{\eta}-u_{\eta, s}\right)-\rho_{\eta} g\right] \cdot \xi_{\sigma} d x=0$.

From Lemma 3.5, there exists $\eta_{0}$ such that $\eta<\eta_{0}$ implies:

$$
\int_{\Omega} H_{\eta} \rho_{\eta}\left(u_{\eta}-u_{\eta, s}\right) \cdot \xi_{\sigma} d x=0
$$

By integration by parts

$$
\begin{gathered}
\int_{\Omega}-2 \mu \operatorname{div}\left(D\left(u_{\eta}\right)\right) \cdot \xi_{\sigma} d x=\int_{\Omega} 2 \mu D\left(u_{\eta}\right): D\left(\xi_{\sigma}\right) d x \\
\int_{\Omega} \operatorname{div}\left(\rho_{\eta} u_{\eta} \otimes u_{\eta}\right) \cdot \xi_{\sigma} d x=\int_{\Omega}-\left(\rho_{\eta} u_{\eta} \otimes u_{\eta}\right): D\left(\xi_{\sigma}\right) d x \\
\int_{\Omega} \frac{\partial\left(\rho_{\eta} u_{\eta}\right)}{\partial t} \cdot \xi_{\sigma} d x=\frac{d}{d t} \int_{\Omega} \rho_{\eta} u_{\eta} \cdot \xi_{\sigma} d x-\int_{\Omega} \rho_{\eta} u_{\eta} \cdot \frac{\partial \xi_{\sigma}}{\partial t} d x .
\end{gathered}
$$

As a result

$$
\int_{\Omega}\left[\rho_{\eta} u_{\eta} \cdot \frac{\partial \xi_{\sigma}}{\partial t}+\left(\rho_{\eta} u_{\eta} \otimes u_{\eta}-2 \mu D\left(u_{\eta}\right)\right): D\left(\xi_{\sigma}\right)+\rho_{\eta} g \cdot \xi_{\sigma}\right] d x=\frac{d}{d t} \int_{\Omega} \rho_{\eta} u_{\eta} \cdot \xi_{\sigma} d x .
$$

We have already established that $u_{\eta} \rightarrow u$ in $L^{2}\left(0, T, H_{0}^{1}(\Omega)\right)$ weak, $u_{\eta} \rightarrow u$ in $L^{2}\left(0, T, L^{2}(\Omega)\right)$ strong, $\rho_{\eta} u_{\eta} \rightarrow \rho u$ in $L^{2}\left(0, T, L^{2}(\Omega)\right)$ weak, and $\rho_{\eta} \rightarrow \rho$ in $L^{2}\left(0, T, L^{2}(\Omega)\right)$ strong. Letting $\eta$ goes to zero, we thus obtain

$$
\int_{\Omega}\left[\rho u \cdot \frac{\partial \xi_{\sigma}}{\partial t}+(\rho u \otimes u-2 \mu D(u)): D\left(\xi_{\sigma}\right)+\rho g \cdot \xi_{\sigma}\right] d x=\frac{d}{d t} \int_{\Omega} \rho u \cdot \xi_{\sigma} d x
$$

which corresponds to the weak formulation (11). This holds for any $\xi_{\sigma} \in H^{1}(Q) \cap L^{2}\left(0, T ; K_{\sigma}^{1}(t)\right)$, for arbitrary $\sigma>0$, and, since the time interval has been chosen to guarantee that there is no contact with the boundary, by Proposition 4.3 of [20], for all $\xi \in H^{1}(Q) \cap L^{2}(0, T ; K(t))$. This ends the proof of theorem 2.1. 


\section{Numerical simulations}

We give here a few numerical illustrations of the penalization method in $2 \mathrm{D}$. We only sketch the numerical discretization and we refer to [3] for a more detailed description and further numerical results.

Numerical 2D and 3D validations against experimental and other numerical results for a discretization of the present penalization method in a vorticity formulation of the Navier-Stokes equations are given in [7].

We choose a time-step $\Delta t$ and denote by a superscript $n$ discretization of all quantities at time $t_{n}=n \Delta t$. For each time integration, we split the penalization model (12)-(16) as follows

- We solve the following variable density flow problem and obtain:

$$
\widetilde{u}=u^{n}-\Delta t\left(u^{n} \cdot \nabla\right) u^{n}+\frac{\Delta t}{\rho^{n}} \mu \Delta \widetilde{u}-\frac{\Delta t}{\rho^{n}} \nabla p^{n+1}+\Delta t g
$$

with

$$
\rho^{n}=\rho_{s} H^{n}+\rho_{f}\left(1-H^{n}\right)
$$

- We compute the rigid velocity the rigid velocity corresponding to $\tilde{u}$ :

$$
u_{s}=u_{G}+w \times r^{n}, \quad u_{G}=\frac{\int_{\Omega} \rho^{n} \widetilde{u} H^{n} d x}{\int_{\Omega} \rho^{n} H^{n} d x}, \quad \omega=J^{-1} \int_{\Omega} \rho^{n}\left(r^{n} \times \widetilde{u}\right) H^{n} d x
$$

- We penalize the flow with this rigid velocity inside the solid body:

$$
\frac{u^{n+1}-\widetilde{u}}{\Delta t}=\frac{1}{\eta} H^{n}\left(u_{s}-u^{n+1}\right) \Leftrightarrow u^{n+1}=\frac{\widetilde{u}+\frac{\Delta t}{\eta} H^{n} u_{s}}{1+\frac{\Delta t}{\eta} H^{n}}
$$

- We finally advect the solid with the rigid velocity:

$$
H^{n+1}=H^{n}-\Delta t u_{s} \cdot \nabla H^{n}
$$

The variable-density flow equations are solved by a projection method using a MAC staggered grid: $p$ and $H$ share the same nodes, while the horizontal and vertical components of velocity are located respectively between a pressure node and its right or upper neighbor. This arrangement of the degrees of freedom ensures an algebraic divergence free vector field during the projection step, and prevents parasitic pressure modes. The advection equation is discretized by a fifth order 
WENO scheme, using the real velocity for the density and the rigid velocity field for $H$, ensuring a constant shape of the rigid body.

Note that we have used an implicit time discretization of the velocity penalization, which allows to use a very small penalization parameter $\eta$. Using an explicit method would require this value no to be smaller than $\Delta t$. It can indeed be checked that a explicit method with $\eta=\Delta t$ essentially amounts to the projection method [18]. We will see below that using smaller values of $\eta$ together with an implicit scheme has a significant effect on the accuracy of the method.

In order to numerically validate the penalization method, we consider the case of the sedimentation of a rigid cylinder in two dimensions (see [11], [7]). The domain $\Omega=[0,2] \times[0,6]$ is filled with an incompressible viscous fluid initially at rest, of density $\rho_{f}=1$ and viscosity $\mu_{f}=0.01$. The rigid cylinder of radius $R=0.125$ and density $\rho_{s}=1.5$ is initially centered in $(1,4)$, and we apply the gravity force $g=-980$. In this experiment, the Reynolds number based on the cylinder diameter varies from 0 to about 250 .

In order to verify how the rigid constraint is satisfied in the solid, we monitor at time $t=0.1$ the $L^{2}$-norm of the discrete deformation tensor defined by:

$$
\|D(u)\|_{L^{2}\left(\Omega_{s}(t)\right)}^{2}:=\sum_{i j} H_{i j}\left(D_{11}^{2}\left(u_{i j}\right)+2 D_{12}^{2}\left(u_{i j}\right)+D_{22}^{2}\left(u_{i j}\right)\right)(\Delta x)^{2}
$$

We fix $\Delta x=1 / 256$ and $\Delta t=10^{-4}$, and compute this norm for values of $\eta$ from $10^{-4}$ to $10^{-12}$, at $t=0.1$.

The results presented in table 1 indicate a convergence of the penalization method, as far as the deformation is concerned, with first order in $\eta$. Note that in our decomposition of the penalization model, we have chosen to verify the divergence free constraint in the stage that precedes the computation of the rigid velocity. As a result, the solution at the end of a complete time step exhibits a boundary layer in the divergence of the velocity, proportional to $\left.\frac{\Delta t}{\eta} \|\left(u_{S}-u\right) \cdot n\right) \|_{L^{2}\left(\partial \Omega_{S}\right)}$. If we had chosen to apply the pressure correction after the equation (54) the divergence of the velocity would be algebraically zero at the end of the time step but the deformation inside the body would be significantly higher.

In figure 1 we show the profiles of the vertical velocity for several values of $\eta$, corresponding to a cross section at the center of the cylinder. We can observe that below $\eta=10^{-8}$ one may consider that we obtained converged velocity results. The projection method of $[18,22]$ essentially consists 


\begin{tabular}{|c|c|c|}
\hline$\eta$ & $\|D(u)\|_{L^{2}\left(\Omega_{s}(t)\right)}$ & $\alpha$ for $O\left(\eta^{\alpha}\right)$ \\
\hline $10^{-4}$ & 4.30838 & - \\
$10^{-6}$ & $3.84749 \times 10^{-2}$ & 1.0247 \\
$10^{-8}$ & $3.45379 \times 10^{-4}$ & 1.0234 \\
$10^{-10}$ & $3.81643 \times 10^{-6}$ & 0.9783 \\
$10^{-12}$ & $3.79832 \times 10^{-8}$ & 1.001 \\
\hline
\end{tabular}

Table 1: Sedimentation of a two dimensional cylinder. Errors on $\|D(u)\|_{L^{2}\left(\Omega_{s}(t)\right)}$ and convergence orders at $t=0.1$ for $\Delta x=1 / 256$ and $\Delta t=10^{-4}$.

of replacing the step (54) by $u_{n+1}=u_{s}$. This corresponds to the following explicit discretization of the penalization term:

$$
\frac{u^{n+1}-\widetilde{u}}{\Delta t}=\frac{1}{\eta} H^{n}\left(u_{s}-\widetilde{u}\right)
$$

with $\Delta t=\eta$. We show the results obtained with these parameters and this discretization of the penalization term. As far as precision is concerned, one can notice the benefit of using larger penalization parameters combined with an implicit time discretization of the penalization term. This confirms the results reported in [7].

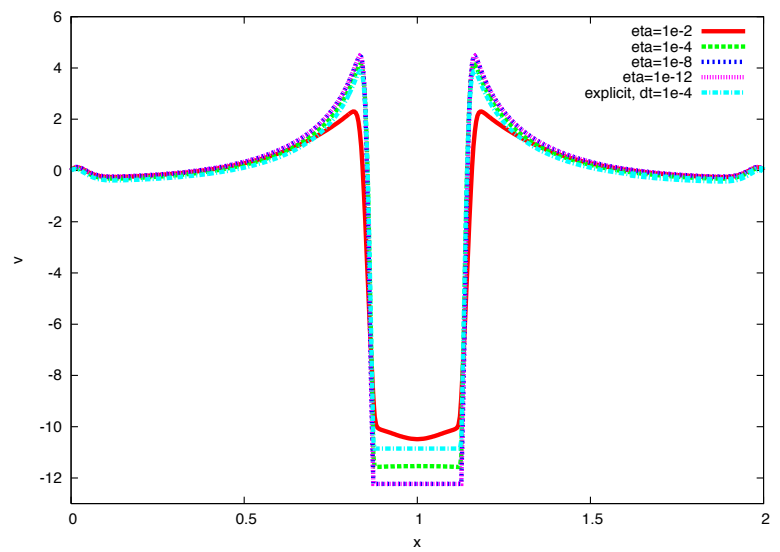

Figure 1: Sedimentation of a two-dimensional cylinder, for $\Delta x=1 / 256$ and $\Delta t=10^{-4}$. Vertical velocity in an horizontal cross-section through the center of the cylinder at $t=0.1$ for several values of the penalization parameter. 


\section{Conclusion}

We have presented and analyzed a penalization method that extends the method of [2] to the case of a rigid body moving freely in an incompressible fluid. The proof is based on compactness arguments. Numerical illustrations have been provided to illustrate our convergence result. The benefit of using very large penalization parameters combined with an implicit time discretization of the penalization term, compared to the projection method [22] which corresponds to a particular explicit time discretization for the penalized equation, has been demonstrated.

While this was not our primary goal, an outcome of our convergence study is an existence result for a weak formulation of the coupling between a rigid solid and a fluid. Let us shortly discuss how this result compares with existing ones $[12,8,6,20]$. In [12] local in time existence and uniqueness of strong solutions was proved. The Eulerian approach was developed in [8] where global in time existence of weak solutions was proved in dimension 2, without collisions. In the three-dimensional case, to our knowledge only local in time existence of weak solutions was obtained, since $L^{2}$ regularity of the time derivative of velocity was required (and therefore global existence would imply global existence of strong solutions). In $[6,13]$ the existence of global weak solution in three dimensions for one ball shaped solid, with possible collision with the boundary, was proved. In [20] the existence of global weak solutions for several rigid bodies with collisions was proved in dimension 2. Our results prove the existence of global in time weak solutions in three

dimensions, before collision. By contrast with $[6,13]$, this result can easily be generalized to the case of several bodies by introducing indicator functions, rigid velocities and penalization terms corresponding to each body. To our knowledge, the existence of global in time weak solutions for several bodies with collisions is an open problem in three dimensions.

\section{Appendix}

This section si devoted to the proof of some technical lemmas that were used in section 3 . 
Proof of Lemma 3.3 Let $\phi(t) \in L^{2}\left(\Omega \backslash \overline{\Omega_{s, \sigma}}(t)\right)$, and $(v(t), q(t)) \in H^{1}\left(\Omega \backslash \overline{\Omega_{s, \sigma}}(t)\right) \times L^{2}\left(\Omega \backslash \overline{\Omega_{s, \sigma}}(t)\right)$ solution of the Stokes problem

$$
\begin{cases}-\Delta v(t)+\nabla q(t)=\phi(t) & \text { on } \Omega \backslash \overline{\Omega_{s, \sigma}}(t), \\ \operatorname{div} v(t)=0 & \text { on } \Omega \backslash \overline{\Omega_{s, \sigma}}(t), \\ v(t)=0 & \text { on } \partial\left(\Omega \backslash \overline{\Omega_{s, \sigma}}(t)\right) .\end{cases}
$$

Since we assumed a $\mathcal{C}^{2}$ regularity on $\Omega \backslash \Omega_{s}^{0}$ this regularity is conserved through rigid motion and, for $\sigma$ small enough (say $\sigma<\sigma_{0}$ for some $\sigma_{0}>0$ ) to $\Omega \backslash \Omega_{s, \sigma}(t)$. The regularity results of AgmonDouglis-Nirenberg on the linear Stokes problem (see [23], prop. 2.3. p. 35) give

$$
(v(t), q(t)) \in H^{2}\left(\Omega \backslash \overline{\Omega_{s, \sigma}}(t)\right) \times H^{1}\left(\Omega \backslash \overline{\Omega_{s, \sigma}}(t)\right)
$$

and there exists $C>0$ such that

$$
\|v(t)\|_{H^{2}\left(\Omega \backslash \overline{\Omega_{s, \sigma}}(t)\right)}+\|q(t)\|_{H^{1}\left(\Omega \backslash \overline{\Omega_{s, \sigma}}(t)\right)} \leq C\|\phi(t)\|_{L^{2}\left(\Omega \backslash \overline{\Omega_{s, \sigma}}(t)\right)} .
$$

Note that, with our definition of $T$, the constant $C$, which depends on the geometry of the domain boundary, can be taken independent of $t \in[0, T]$ and $\sigma$, provided $\sigma_{0}$ is taken small enough. We can then write

$$
\begin{aligned}
\int_{\Omega \backslash \overline{\Omega_{s, \sigma}}(t)} w(t) \cdot \phi(t) d x= & -\int_{\Omega \backslash \overline{\Omega_{s, \sigma}}(t)} w(t) \cdot \Delta v(t) d x+\int_{\Omega \backslash \overline{\Omega_{s, \sigma}}(t)} w(t) \cdot \nabla q(t) d x \\
= & -\int_{\partial\left(\Omega \backslash \overline{\Omega_{s, \sigma}}\right)(t)} w(t) \cdot \frac{\partial v(t)}{\partial n} d s+\int_{\Omega \backslash \overline{\Omega_{s, \sigma}}(t)} \nabla w(t) \cdot \nabla v(t) d x \\
& +\int_{\partial\left(\Omega \backslash \overline{\Omega_{s, \sigma}}\right)(t)} w(t) q(t) \cdot n d s-\int_{\Omega \overline{\Omega_{s, \sigma}}(t)} \operatorname{div} w(t) q(t) d x \\
= & -\int_{\partial \Omega_{s, \sigma}(t)} w(t) \cdot \frac{\partial v(t)}{\partial n} d s+\int_{\partial\left(\Omega \backslash \overline{\Omega_{s, \sigma}}\right)(t)} \frac{\partial w(t)}{\partial n} \cdot v(t) d s \\
& -\int_{\Omega \backslash \overline{\Omega_{s, \sigma}}(t)} \Delta w(t) \cdot v(t) d x+\int_{\partial \Omega_{s, \sigma}(t)} w(t) q(t) \cdot n d s \\
= & -\int_{\partial \Omega_{s, \sigma}(t)} w(t) \cdot \frac{\partial v(t)}{\partial n} d s+\int_{\Omega \backslash \overline{\Omega_{s, \sigma}}(t)} v(t) \cdot \nabla p(t) d x \\
& +\int_{\partial \Omega_{s, \sigma}(t)} w(t) q(t) \cdot n d s
\end{aligned}
$$


The integral of $v \cdot \nabla p$ vanishes since $v$ is divergence-free and vanishes on $\partial\left(\Omega \backslash \overline{\Omega_{s, \sigma}}(t)\right)$. Then using classical trace theorems in Sobolev spaces we get

$$
\begin{aligned}
\int_{\Omega \backslash \overline{\Omega_{s, \sigma}}(t)} w(t) \cdot \phi(t) d x & =-\int_{\partial \Omega_{s, \sigma}(t)} g(t) \cdot \frac{\partial v(t)}{\partial n} d s+\int_{\partial \Omega_{s, \sigma}(t)} g(t) q(t) \cdot n d s \\
& \leq\|g(t)\|_{L^{2}\left(\partial \Omega_{s, \sigma}(t)\right)}\left(\|\nabla v(t)\|_{L^{2}\left(\partial \Omega_{s, \sigma}(t)\right)}+\|q(t)\|_{L^{2}\left(\partial \Omega_{s, \sigma}(t)\right)}\right) \\
& \leq C\|g(t)\|_{L^{2}\left(\partial \Omega_{s, \sigma}(t)\right)}\left(\|v(t)\|_{H^{2}\left(\Omega \backslash \overline{\Omega_{s, \sigma}}(t)\right)}+\|q(t)\|_{H^{1}\left(\Omega \backslash \overline{\Omega_{s, \sigma}}(t)\right)}\right) \\
& \leq C\|g(t)\|_{L^{2}\left(\partial \Omega_{s, \sigma}(t)\right)}\|\phi(t)\|_{L^{2}\left(\Omega \backslash \overline{\Omega_{s, \sigma}}(t)\right)} \\
& \leq C\|u(t)\|_{L^{2}\left(\Omega_{s, \sigma}(t)\right)}^{\frac{1}{2}}\|\nabla u(t)\|_{L^{2}\left(\Omega_{s, \sigma}(t)\right)}^{\frac{1}{2}}\|\phi(t)\|_{L^{2}\left(\Omega \backslash \overline{\Omega_{s, \sigma}}(t)\right)} .
\end{aligned}
$$

This proves the assertion.

\section{Proof of Lemma 3.6}

Step 1: We first show how to construct for a.e. $t \in] 0, T\left[\right.$ a function $v_{\sigma}(., t) \in \mathcal{K}_{\sigma}^{r}(t)$ such that

$$
\left.\lim _{\sigma \rightarrow 0}\left\|v_{\sigma}(., t)-u(., t)\right\|_{\mathcal{V}^{r}}=0 \text { a.e. on }\right] 0, T[.
$$

Let $\sigma>0$ and $v_{\sigma}(., t)$ such that

$$
\begin{cases}-\Delta v_{\sigma}(., t)+\nabla p(., t)=-\Delta u(., t) & \text { on } \Omega \backslash \overline{\Omega_{s, \sigma}}(t), \\ \operatorname{div} v_{\sigma}(., t)=0 & \text { on } \Omega \backslash \overline{\Omega_{s, \sigma}}(t), \\ v_{\sigma}(., t)=u_{s}(., t) & \text { on } \partial \Omega_{s, \sigma}(t), \\ v_{\sigma}(., t)=0 & \text { on } \partial \Omega,\end{cases}
$$

where

$$
u_{s}=\frac{1}{M} \int_{\Omega} \rho u H d x+\left(J^{-1} \int_{\Omega} \rho(r \times u) H d x\right) \times r .
$$

By lemma 3.4, $u(., t)=u_{s}(., t)$ on $\Omega_{s}(t)$. Extending $v_{\sigma}(., t)$ by $u_{s}(., t)$ in $\Omega_{s, \sigma}(t)$, we have $v_{\sigma}(., t) \in$ $\mathcal{K}_{\sigma}^{r}(t)$. We set $e_{\sigma}(., t)=v_{\sigma}(., t)-u(., t)$. It satisfies

$$
\begin{cases}-\Delta e_{\sigma}(., t)+\nabla p(., t)=0 & \text { on } \Omega \backslash \overline{\Omega_{s, \sigma}}(t), \\ \operatorname{div} e_{\sigma}(., t)=0 & \text { on } \Omega \backslash \overline{\Omega_{s, \sigma}}(t), \\ e_{\sigma}(., t)=u_{s}(., t)-u(., t) & \text { on } \partial \Omega_{s, \sigma}(t), \\ e_{\sigma}(., t)=0 & \text { on } \partial \Omega .\end{cases}
$$

We extend $e_{\sigma}(., t)$ by $u_{s}(., t)-u(., t)$ in $\Omega_{s, \sigma}(t)$, so that $e_{\sigma}(., t)=0$ in $\Omega_{s}(t)$.

We claim that

$$
\left.\lim _{\sigma \rightarrow 0}\left\|e_{\sigma}(., t)\right\|_{L^{2}(\Omega)}=0 \text { a.e. on }\right] 0, T[.
$$


In $\Omega_{s}(t) e_{\sigma}(., t)=0$, thus

$$
\left\|e_{\sigma}(., t)\right\|_{L^{2}(\Omega)}^{2}=\left\|e_{\sigma}(., t)\right\|_{L^{2}\left(\Omega_{s, \sigma}(t) \backslash \overline{\Omega_{s}}(t)\right)}^{2}+\left\|e_{\sigma}(., t)\right\|_{L^{2}\left(\Omega \backslash \overline{\Omega_{s, \sigma}}(t)\right)}^{2} .
$$

Since $\Omega_{s, \sigma}(t) \backslash \overline{\Omega_{s}}(t)$ has width $2 \sigma$, from the proof of lemma 5.10 of [10] we have a.e. on ]0,T[,

$$
\begin{aligned}
\left\|e_{\sigma}(., t)\right\|_{L^{2}\left(\Omega_{s, \sigma}(t) \backslash \overline{\Omega_{s}}(t)\right)}^{2} & \leq C\left(\left\|e_{\sigma}(., t)\right\|_{L^{2}\left(\partial \Omega_{s}(t)\right)}^{2}+\sigma^{2}\left\|\nabla e_{\sigma}(., t)\right\|_{L^{2}\left(\Omega_{s, \sigma}(t) \backslash \overline{\Omega_{s}}(t)\right)}^{2}\right) \\
& \leq C\left(\left\|e_{\sigma}(., t)\right\|_{L^{2}\left(\Omega_{s}(t)\right)}\left\|\nabla e_{\sigma}(., t)\right\|_{L^{2}\left(\Omega_{s}(t)\right)}+\sigma^{2}\left\|\nabla e_{\sigma}(., t)\right\|_{L^{2}\left(\Omega_{s, \sigma}(t) \backslash \overline{\Omega_{s}}(t)\right)}^{2}\right) \\
& =C \sigma^{2}\left\|\nabla e_{\sigma}(., t)\right\|_{L^{2}\left(\Omega_{s, \sigma}(t) \backslash \overline{\Omega_{s}}(t)\right)}^{2}
\end{aligned}
$$

Next, as $e_{\sigma}(., t)=u_{s}(., t)-u(., t)$ in $\Omega_{s, \sigma}(t)$ and $u(., t)$ and $u_{s}(., t)$ are in $H_{0}^{1}(\Omega)$, we get $\left\|\nabla e_{\sigma}(., t)\right\|_{L^{2}\left(\Omega_{s, \sigma}(t) \backslash \overline{\Omega_{s}}(t)\right)} \leq$ $\left\|\nabla e_{\sigma}(., t)\right\|_{L^{2}\left(\Omega_{s, \sigma}(t)\right)} \leq\left\|\nabla e_{\sigma}(., t)\right\|_{L^{2}(\Omega)} \leq C$, where $C$ is independent of $\sigma$. This gives

$$
\left\|e_{\sigma}(., t)\right\|_{L^{2}\left(\Omega_{s, \sigma}(t)\right)}=\left\|e_{\sigma}(., t)\right\|_{L^{2}\left(\Omega_{s, \sigma}(t) \backslash \overline{\Omega_{s}}(t)\right)} \leq C \sigma .
$$

By Lemma 3.3 we thus get

$$
\left\|e_{\sigma}(., t)\right\|_{L^{2}\left(\Omega \backslash \overline{\Omega_{s, \sigma}}(t)\right)}^{2} \leq C\left\|e_{\sigma}(., t)\right\|_{L^{2}\left(\Omega_{s, \sigma}(t)\right)}\left\|\nabla e_{\sigma}(., t)\right\|_{L^{2}\left(\Omega_{s, \sigma}(t)\right)} \leq C \sigma .
$$

Collecting the above estimates, we conclude that

$$
\lim _{\sigma \rightarrow 0}\left\|e_{\sigma}(., t)\right\|_{L^{2}(\Omega)}^{2}=0 .
$$

In order to prove that this convergence also holds in $\mathcal{V}^{r}$ we first note that

$$
\left.\left\|e_{\sigma}(., t)\right\|_{H^{1}(\Omega)} \leq C \text { a.e. on }\right] 0, T[
$$

as is readily seen from estimates on the Stokes problem verified by $e_{\sigma}$. By interpolation (see e.g. [1], p. 135), we obtain

$$
\left\|e_{\sigma}(., t)\right\|_{\mathcal{V}^{r}} \leq\left\|e_{\sigma}(., t)\right\|_{L^{2}(\Omega)}^{1-r}\left\|e_{\sigma}(., t)\right\|_{H^{1}(\Omega)}^{r}
$$

and due to (55) and (56),

$$
\lim _{\sigma \rightarrow 0}\left\|e_{\sigma}(., t)\right\|_{\mathcal{V}^{r}}=0 \forall r \in[1 / 2,1[\text { a.e. on }] 0, T[.
$$

Step 2: By definition of $P_{\sigma}^{r}$,

$$
\left\|P_{\sigma}^{r} u(., t)-u(., t)\right\|_{\mathcal{V}^{r}} \leq\left\|v_{\sigma}(., t)-u(., t)\right\|_{\mathcal{V}^{r}}
$$


thus the pointwise convergence on $v_{\sigma}$ we just obtained implies

$$
\left.\lim _{\sigma \rightarrow 0}\left\|P_{\sigma}^{r} u(., t)-u(., t)\right\|_{\mathcal{V}^{r}}=0 \text { a.e. on }\right] 0, T[\text {. }
$$

Step 3: $f_{\sigma}: t \mapsto\left\|P_{\sigma}^{r} u(., t)-u(., t)\right\|_{\mathcal{V}^{r}}$ is measurable on $[0, T]$ and since $0 \in \mathcal{K}_{\sigma}^{r}$,

$$
\begin{aligned}
\left\|f_{\sigma}\right\|_{L^{\frac{2}{r}(0, T)}}^{\frac{2}{r}}=\int_{0}^{T}\left\|P_{\sigma}^{r} u(., t)-u(., t)\right\|_{\mathcal{V}^{r}}^{\frac{2}{r}} d t & \leq \int_{0}^{T}\|u(., t)\|_{\mathcal{V}^{r}}^{\frac{2}{r}} d t \\
& \leq C \int_{0}^{T}\|u(., t)\|_{L^{2}(\Omega)}^{\frac{2(1-r)}{r}}\|u(., t)\|_{H^{1}(\Omega)}^{2} d t \\
& \leq C\|u\|_{L^{\infty}\left(0, T, L^{2}(\Omega)\right)}^{\frac{2(1-r)}{r}}\|u\|_{L^{2}\left(0, T, H_{0}^{1}(\Omega)\right)}^{2} \\
& \leq C .
\end{aligned}
$$

To summarize $f_{\sigma}$ verifies

$$
\left\{\begin{array}{l}
\lim _{\sigma \rightarrow 0} f_{\sigma}(t)=0 \text { a.e. on }[0, T], \\
f_{\sigma} \text { is measurable on }[0, T], \\
\left\|f_{\sigma}\right\|_{L^{\frac{2}{r}}(0, T)} \leq C \text { with } r<1 .
\end{array}\right.
$$

Therefore, thanks to lemma $3.2, \lim _{\sigma \rightarrow 0}\left\|f_{\sigma}\right\|_{L^{2}(0, T)}=0$, which means

$$
\lim _{\sigma \rightarrow 0}\left\|P_{\sigma}^{r} u-u\right\|_{L^{2}\left(0, T, \mathcal{V}^{r}\right)}=0 .
$$

\section{Proof of Lemma 3.7}

Step 1: We construct for a.e. fixed $t \in[0, T]$ a function $v_{\eta \sigma}(., t) \in \mathcal{K}_{\sigma}^{r}(t)$ such that

$$
\left.\lim _{\sigma \rightarrow 0} \lim _{\eta \rightarrow 0}\left\|v_{\eta \sigma}(., t)-u_{\eta}(., t)\right\|_{\mathcal{V}^{r}}=0 \text { a.e. on }\right] 0, T[.
$$

Let $\sigma>0$ and $v_{\eta \sigma}(., t)$ solution of the following Stokes problem outside $\Omega_{s, \sigma}^{\eta}(t)$ :

$$
\begin{cases}-\Delta v_{\eta \sigma}(., t)+\nabla p(., t)=-\Delta u_{\eta}(., t) & \text { on } \Omega \backslash \overline{\Omega_{s, \sigma}^{\eta}}(t), \\ \operatorname{div} v_{\eta \sigma}(., t)=0 & \text { on } \Omega \backslash \overline{\Omega_{s, \sigma}^{\eta}}(t), \\ v_{\eta \sigma}(., t)=u_{\eta, s}(., t) & \text { on } \partial \Omega_{s, \sigma}^{\eta}(t), \\ v_{\eta \sigma}(., t)=0 & \text { on } \partial \Omega .\end{cases}
$$

Extending $v_{\eta \sigma}(., t)$ by $u_{\eta, s}(., t)$ in $\Omega_{s, \sigma}^{\eta}(t)$, we have $v_{\eta \sigma}(., t) \in \mathcal{K}_{\sigma}^{r}(t)$. We then introduce $e_{\eta \sigma}(., t)=$ $v_{\eta \sigma}(., t)-u_{\eta}(., t)$. It verifies

$$
\begin{cases}-\Delta e_{\eta \sigma}(., t)+\nabla p(., t)=0 & \text { on } \Omega \backslash \overline{\Omega_{s, \sigma}^{\eta}}(t), \\ \operatorname{div} e_{\eta \sigma}(., t)=0 & \text { on } \Omega \backslash \overline{\Omega_{s, \sigma}^{\eta}}(t), \\ e_{\eta \sigma}(., t)=u_{\eta, s}(., t)-u_{\eta}(., t) & \text { on } \partial \Omega_{s, \sigma}^{\eta}(t), \\ e_{\eta \sigma}(., t)=0 & \text { on } \partial \Omega\end{cases}
$$

and we extend it by $u_{\eta, s}(., t)-u_{\eta}(., t)$ in $\Omega_{s, \sigma}^{\eta}(t)$. 
We claim that

$$
\left.\lim _{\sigma \rightarrow 0} \lim _{\eta \rightarrow 0}\left\|e_{\eta \sigma}(., t)\right\|_{L^{2}(\Omega)}=0 \text { a.e. on }\right] 0, T[.
$$

From lemma 3.5, for a given $\sigma>0$, there exists $\eta_{0}>0$ such that $\forall \eta<\eta_{0}$,

$$
\Omega_{s}^{\eta}(t) \subset \Omega_{s, \sigma}(t) \text { and } \Omega_{s}(t) \subset \Omega_{s, \sigma}^{\eta}(t) .
$$

Let $\eta<\eta_{0}$. We write

$$
\left\|e_{\eta \sigma}(., t)\right\|_{L^{2}(\Omega)}^{2}=\left\|e_{\eta \sigma}(., t)\right\|_{L^{2}\left(\Omega_{s}^{\eta}(t)\right)}^{2}+\left\|e_{\eta \sigma}(., t)\right\|_{L^{2}\left(\Omega_{s, \sigma}(t) \backslash \overline{\Omega_{s}^{\eta}}(t)\right)}^{2}+\left\|e_{\eta \sigma}(., t)\right\|_{L^{2}\left(\Omega \backslash \overline{\Omega_{s, \sigma}}(t)\right)}^{2} .
$$

From estimate (23), there holds

$$
\int_{0}^{T}\left\|e_{\eta \sigma}(., t)\right\|_{L^{2}\left(\Omega_{s}^{\eta}(t)\right)}^{2} d t \leq C \eta
$$

Since $\Omega_{s, \sigma}(t) \backslash \overline{\Omega_{s}^{\eta}}(t)$ has width less than $2 \sigma$, from the proof of lemma 5.10 of [10] we have a.e. on ] $0, T[$,

$$
\left\|e_{\eta \sigma}(., t)\right\|_{L^{2}\left(\Omega_{s, \sigma}(t) \backslash \overline{\Omega_{s}^{\eta}}(t)\right)}^{2} \leq C\left(\left\|e_{\eta \sigma}(., t)\right\|_{L^{2}\left(\partial \Omega_{s}^{\eta}(t)\right)}^{2}+\sigma^{2}\left\|\nabla e_{\eta \sigma}(., t)\right\|_{L^{2}\left(\Omega_{s, \sigma}(t) \backslash \overline{\Omega_{s}^{\eta}}(t)\right)}^{2}\right) .
$$

And using a trace theorem, we get

$$
\begin{aligned}
\left\|e_{\eta \sigma}(., t)\right\|_{L^{2}\left(\Omega_{s, \sigma}(t) \backslash \overline{\Omega_{s}^{\eta}}(t)\right)}^{2} \leq C\left(\left\|e_{\eta \sigma}(., t)\right\|_{L^{2}\left(\Omega_{s}^{\eta}(t)\right)}\left\|\nabla e_{\eta \sigma}(., t)\right\|_{L^{2}\left(\Omega_{s}^{\eta}(t)\right)}\right. & \left.+\sigma^{2}\left\|\nabla e_{\eta \sigma}(., t)\right\|_{L^{2}\left(\Omega_{s, \sigma}(t) \backslash \overline{\Omega_{s}^{\eta}}(t)\right)}^{2}\right) \\
\leq C\left(\left\|e_{\eta \sigma}(., t)\right\|_{L^{2}\left(\Omega_{s}^{\eta}(t)\right)}\left\|\nabla e_{\eta \sigma}(., t)\right\|_{L^{2}\left(\Omega_{s, \sigma}(t)\right)}\right. & \left.+\sigma^{2}\left\|\nabla e_{\eta \sigma}(., t)\right\|_{L^{2}\left(\Omega_{s, \sigma}(t)\right)}^{2}\right) .
\end{aligned}
$$

Adding $\left\|e_{\eta \sigma}(., t)\right\|_{L^{2}\left(\Omega_{s}^{\eta}(t)\right)}^{2}$ to this inequality gives

$$
\begin{array}{r}
\left\|e_{\eta \sigma}(., t)\right\|_{L^{2}\left(\Omega_{s, \sigma}(t)\right)}^{2} \leq C\left(\left\|e_{\eta \sigma}(., t)\right\|_{L^{2}\left(\Omega_{s}^{\eta}(t)\right)}^{2}+\left\|e_{\eta \sigma}(., t)\right\|_{L^{2}\left(\Omega_{s}^{\eta}(t)\right)}\left\|\nabla e_{\eta \sigma}(., t)\right\|_{L^{2}\left(\Omega_{s, \sigma}(t)\right)}\right. \\
\left.+\sigma^{2}\left\|\nabla e_{\eta \sigma}(., t)\right\|_{L^{2}\left(\Omega_{s, \sigma}(t)\right)}^{2}\right) .
\end{array}
$$

For the last term in (61) we use Lemma 3.3:

$$
\left\|e_{\eta \sigma}(., t)\right\|_{L^{2}\left(\Omega \backslash \overline{\Omega_{s, \sigma}}(t)\right)}^{2} \leq C\left\|e_{\eta \sigma}(., t)\right\|_{L^{2}\left(\Omega_{s, \sigma}(t)\right)}\left\|\nabla e_{\eta \sigma}(., t)\right\|_{L^{2}\left(\Omega_{s, \sigma}(t)\right)} .
$$


Since $e_{\eta \sigma}(., t)=u_{\eta, s}(., t)-u_{\eta}(., t)$ in $\Omega_{s, \sigma}(t)$ and $u_{\eta}, u_{\eta, s}$ are bounded in $L^{2}\left(0, T ; H_{0}^{1}(\Omega)\right)$, we have

$$
\int_{0}^{T}\left\|\nabla e_{\eta \sigma}(., t)\right\|_{L^{2}\left(\Omega_{s, \sigma}(t)\right)}^{2} d t \leq C .
$$

With (62) and (67) we are now in position to estimate the integral over $[0, T]$ of (64-66). By

Cauchy-Schwarz inequality:

$$
\begin{gathered}
\int_{0}^{T}\left\|e_{\eta \sigma}(., t)\right\|_{L^{2}\left(\Omega_{s, \sigma}(t) \backslash \overline{\Omega_{s}^{\eta}}(t)\right)}^{2} d t \leq C\left(\eta^{\frac{1}{2}}+\sigma^{2}\right), \\
\int_{0}^{T}\left\|e_{\eta \sigma}(., t)\right\|_{L^{2}\left(\Omega \backslash \overline{\Omega_{s, \sigma}}(t)\right)}^{2} d t \leq C\left(\int_{0}^{T}\left\|e_{\eta \sigma}(., t)\right\|_{L^{2}\left(\Omega_{s, \sigma}(t)\right)}^{2} d t\right)^{\frac{1}{2}} \leq C\left(\eta+\eta^{\frac{1}{2}}+\sigma^{2}\right)^{\frac{1}{2}} .
\end{gathered}
$$

Therefore, for a fixed value of $\sigma$ we can pass to the limit in $\eta$, and then pass to the limit in $\sigma$, to obtain

$$
\lim _{\sigma \rightarrow 0} \lim _{\eta \rightarrow 0} \int_{0}^{T}\left\|e_{\eta \sigma}(., t)\right\|_{L^{2}(\Omega)}^{2} d t=0 .
$$

This strong convergence can be turned into an almost everywhere in $t$ convergence up to the extraction of a subsequence. The rest of the proof is adapted in a straightforward way from that of Lemma 3.6.

\section{References}

[1] R.A. Adams and J. Fournier, Sobolev spaces, second edition, Elsevier (2003)

[2] Ph. Angot, C.-H. Bruneau and P. Fabrie, A penalization method to take into account obstacles in incompressible viscous flows, Numer. Math. 81: 497-520 (1999)

[3] C. Bost, Méthodes Level-Set et pénalisation pour le calcul d'interactions fluide-structure, PhD Thesis, University of Grenoble, France (2008). Available for download at http://tel. archives-ouvertes.fr/tel-00341209/fr/.

[4] F. Boyer and P. Fabrie, Eléments d'analyse pour l'étude de quelques modèles d'écoulements de fluides visqueux incompressibles, Mathématiques \& Applications 52 (2006)

[5] H. Brezis. Analyse fonctionnelle, Théorie et applications, Masson (1992).

[6] C. Conca, H.J. San Martín and M. Tucsnak, Existence of solutions for the equations modelling the motion of a rigid body in a viscous fluid, Comm. Partial Differential Equations 25, 1019$1042(2000)$

[7] M. Coquerelle and G.-H. Cottet, A vortex level-set method for the two-way coupling of an incompressible fluid with colliding rigid bodies, Journal of Computational Physics, 227, 9121$9137(2008)$

[8] B. Desjardins and M.J. Esteban, Existence of Weak Solutions for the Motion of Rigid Bodies in a Viscous Fluid, Arch. Rational Mech. Anal. 146, 59-71 (1999)

[9] R.J. Di Perna and P-L. Lions, Ordinary differential equations, transport theory and Sobolev spaces, Invent. math. 98, 511-547 (1989) 
[10] H. Fujita and N. Sauer, On existence of weak solutions of the Navier-Stokes equations in regions with moving boundaries, J. Fac. Sci. Univ. Tokyo Sec. 1A (became from 1993 Journal of mathematical sciences, the University of Tokyo), 17, 403-420 (1970)

[11] R. Glowinski, T. W. Pan, T. I. Hesla, D. D. Joseph, and J. Périaux, A Fictitious Domain Approach to the Direct Numerical Simulation of Incompressible Viscous Flow past Moving Rigid Bodies: Application to Particulate Flow, Journal of Computational Physics 169, 363$426(2001)$

[12] C. Grandmont and Y. Maday, Existence de solutions d'un problème de couplage fluidestructure bidimensionel instationnaire, C. R. Acad. Sci Paris Sér. I Math. 326, 525-530 (1998)

[13] M.D. Gunzburger, H.-C. Lee and G.A. Seregin, Global Existence of Weak Solutions for Viscous Incompressible Flows around a Moving Rigid Body in Three Dimensions, J. math. fluid mech. 2, 219-266 (2000)

[14] M. Hillairet, Lack of collision between solid bodies in a $2 D$ constant density incompressible viscous flow, Communications in Partial Differential Equations, 32:9, 1345-1371 (2007)

[15] J. Janela, A. Lefebvre and B. Maury, A Penalty Method for the simulation of fluid-body rigid interaction, ESAIM: Proceedings, Vol.14, 115-123 (2005)

[16] J.-L. Lions, Quelques méthodes de résolutions des problèmes aux limites non linéaires, Dunod (1968)

[17] B. Maury, Direct simulations of $2 D$ fluid-particle flows in biperiodic domains, J. Comput. Phys., 156, pp. 325-351 (1999)

[18] N.A. Patankar, A formulation for fast computations of rigid particulate flows, Center Turbul. Res., Ann. Res. Briefs, 185-196 (2001)

[19] J. San Martín, J.-F. Scheid, T. Takahashi, and M. Tucsnak, Convergence of the LagrangeGalerkin method for the equations modelling the motion of a fluid-rigid system, SIAM J. Numer. Anal., 43 (2005), pp. 1536-1571.

[20] J.A. San Martin, V. Starovoitov and M. Tucsnak. Global Weak Solutions for the TwoDimensional Motion of Several Rigid Bodies in an Incompressible Viscous Fluid, Arch. Rational Mech. Anal. 161, 113-147 (2002)

[21] A. Sarthou, S. Vincent, JP. Caltagirone and Ph. Angot, Eulerian-Lagrangian grid coupling and penalty methods for the simulation of multiphase flows interacting with complex objects, Int. J. Numer. Meth. Fluids 00:1-6 (2007)

[22] N. Sharma and N.A. Patankar, A fast computation technique for the direct numerical simulation of rigid particulate flows, Journal of Computational Physics 205, 439-457 (2005)

[23] R. Temam, Navier-Stokes equations and numerical analysis, North-Holland, Amsterdam (1979) 